\title{
Lügner haben kurze Beine. Zum Zusammenhang unwahrer Selbstdarstellung und partnerschaftlicher Chancen im Online-Dating ${ }^{1}$
}

\author{
Liars have short legs. \\ The relationship between deceptive self-presentation and mating \\ opportunities in online dating
}

\begin{abstract}
Zusammenfassung:
Unwahrheiten sind alltägliche Bestandteile sozialer Interaktionen. Sie treten im Alltag jedoch meist nur in Form kleinerer Lügen und „Schummeleien", wie es umgangssprachlich heißt, auf. Ein Ziel von Unwahrheiten kann sein, sich eine bessere Position zu verschaffen, um daraus Vorteile beispielsweise in Form sozialer Wertschätzung durch Andere zu erhalten. Der vorliegende Beitrag untersucht Unwahrheiten bei der Selbstdarstellung im Kontext der Partnerwahl auf Online-Kontaktbörsen. Mithilfe von Daten aus einer Online-Befragung von Nutzern einer Online-Kontaktbörse wird untersucht, ob Muster unwahrer Selbstdarstellung im Nutzerprofil beobachtbar sind und wie die Merkmale der Akteure die beobachtbaren Muster der Unwahrheit beeinflussen. Dazu führen wir eine analytische Unterscheidung von spezifischer und unspezifischer Kompensation nachteiliger partnerschaftlicher Chancen ein. Die empirischen Analysen konzentrieren sich auf unwahre Profildarstellungen des Bildungsniveaus und der physischen Attraktivität. Die Ergebnisse zeigen deutliche geschlechts- und merkmalsspezifische Muster unwahrer Selbstdarstellung. So zeigt sich beispielsweise hinsichtlich der Körpergröße
\end{abstract}

\begin{abstract}
:
Untruthfulness and deception are common constituents of social interactions. In everyday life, however, they usually occur in form of small lies and cheating. Gaining a better position in order to receive social approval by others might be the intention underlying untrue statements. In this contribution, we investigate deception in selfpresentation in the context of mate choice on online dating sites. Using data from an online survey conducted amongst users of an online dating site, we analyse whether patterns of misrepresentation in users' profiles can be detected and which of the actors' characteristics influence the observable patterns of untruthfulness. For this purpose, we introduce an analytical distinction between specific and unspecific compensation of disadvantageous chances in mating. The empirical analyses focus on deceptive presentation in the user profile concerning educational level and physical attractiveness. The results show clear gender specific and trait specific patterns of deceptive selfpresentation. With regard to, for example, body height, there is a significant effect for men, but not for women: especially small men have a higher probability to misrepresent their height in
\end{abstract}

1 Für wertvolle Hinweise danken wir Jan Skopek, Sebastian E. Wenz, Thorsten Schneider, Knut Wenzig und Stephanie Wenisch. 
ein signifikanter Effekt bei Männern, nicht jedoch bei Frauen: Es sind vor allem kleine Männer, die eine höhere Wahrscheinlichkeit haben, ihre Körpergröße im Nutzerprofil unwahr darzustellen. Diese Strategie bezeichnen wir als spezifische Kompensation nachteiliger Chancen am Partnermarkt. Die Analysen bestätigen aber auch unsere Erwartung hinsichtlich Strategien der unspezifischen Kompensation: tatsächliche oder vermeintliche Nachteile in einem Merkmal können dazu führen, dass sich Akteure in einem anderen Merkmal unwahr darstellen, ebenso können Nachteile in einem Merkmal durch Stärken in anderen Merkmalen kompensiert werden. Mit Hilfe der unspezifischen Kompensation ist es möglich, Unwahrheiten in einem Merkmal gering und damit auch verzeihlich zu halten.

Schlagwörter: Selbstdarstellung, Täuschung, Vertrauen, Informationen, Partnersuche im Internet, Partnerwahl, Online-Dating the user profile. This strategy will be called specific compensation of disadvantageous chances on the partner market. In addition, the analyses support our expectations concerning strategies of unspecific compensation: actual or perceived disadvantages in one characteristic can lead actors to misrepresent themselves in another characteristic; likewise, disadvantages in one characteristic can be compensated by advantages in other characteristics. Using unspecific compensation, actors are able to keep deception in one characteristic on a minimal and therefore excusable level.

Key words: self-presentation, deception, trust, information, mate search on the internet, mate choice, online dating

\section{Einleitung}

Das Internet bietet seit einigen Jahren neue Gelegenheiten zur Partnersuche und hat sich mittlerweile zu einem bedeutenden Partnermarkt entwickelt (vgl. z.B. Lea/Spears 1995; Merkle/Richardson 2000; Ben-Ze'ev 2004; Schulz/Zillmann 2009; Hogan et al. 2011). Es eröffnet verschiedene Möglichkeiten der (virtuellen) Begegnung, des Kennenlernens und der Kommunikation. Als spezielle Orte der Partnersuche haben sich Online-Kontaktbörsen herausgebildet, die sich von anderen so genannten Social Network-Seiten dadurch unterscheiden, dass sie explizit auf die Partnersuche oder Partnervermittlung ausgerichtet sind.

Um die Möglichkeiten zur Partnersuche, die Online-Kontaktbörsen bieten, nutzen zu können, müssen sich die Nutzer zunächst auf der Plattform registrieren. Im Zuge dessen gibt sich jeder Nutzer ein selbst gewähltes Pseudonym, das seine eindeutige Identifizierung auf der Plattform ermöglicht, jedoch nicht seine tatsächliche Identität preisgibt. Im Anschluss daran füllen die Nutzer ein Nutzerprofil aus, mittels dessen sie anderen Nutzern Informationen über sich, beispielsweise zum Alter, Familienstand, Bildungsniveau, zur beruflichen Situation und zum Aussehen bereitstellen. Neben diesen standardisierten Informationen haben die Nutzer die Möglichkeit, ihre Selbstdarstellung im Rahmen des Nutzerprofils über Freitextfelder zu ergänzen und damit ihr Online-Profil individueller und umfangreicher zu gestalten. Zudem können die Nutzer ihr Nutzerprofil mit einem oder mehreren Fotos von sich anreichern, um diesen Steckbrief persönlicher und authentischer zu gestalten.

Diese im Nutzerprofil bereitgestellten Informationen über die eigene Person sind zugleich für die anderen Nutzer der Plattform der wesentliche Ausgangspunkt für die Suche 
und Selektion potenzieller Partner, die dann über plattforminterne Nachrichtensysteme, in der Regel sind das E-Mail-Nachrichten, mitunter auch Chats, kontaktiert werden können. Bei der Selbstdarstellung in einem Nutzerprofil, aber auch in der sich daran anschließenden Kommunikation über E-Mail-Nachrichten oder ein Chatsystem, lassen sich daher zwei idealtypische Ziele benennen. Zum einen möchten Nutzer durch ihre (Profil-)Darstellung ein Höchstmaß an Aufmerksamkeit bei potenziellen Partnern wecken, indem sie sich interessant und attraktiv darstellen (vgl. z.B. Ellison et al. 2006). Denn damit steigt die Chance, mit potenziellen Partnern überhaupt in Kontakt zu kommen und ein Untergehen in der Masse der Partnersuchenden zu verhindern. Zum anderen möchten die meisten Nutzer einer Online-Kontaktbörse eine (kurz- oder langfristige) Partnerschaft verwirklichen. Ein potenzieller Partner erwartet jedoch im Fall einer persönlichen Begegnung auf eine Person zu treffen, die den auf der Kontaktbörse offenbarten Merkmalen entspricht. Die Herausforderung bei der Selbstdarstellung besteht also darin, dass sich Nutzer einerseits optimal darstellen müssen, um ihre Aufmerksamkeits- und damit Kontaktchancen zu erhöhen (vgl. Schmitz 2010). Andererseits macht aber unter Umständen gerade diese Optimierung Anschlussinteraktionen und damit das letztliche Ziel der Etablierung einer Paarbeziehung im (nicht-virtuellen) Alltag unwahrscheinlicher. Das Ziel, eine hohe Aufmerksamkeit durch eine attraktive Profildarstellung zu erreichen, konfligiert also unter Umständen mit dem eigentlichen Ziel, einen Partner zu finden.

Aus der Perspektive der anderen Nutzer ist das Nutzerprofil die wesentliche und zugleich vorerst auch die einzige Informationsquelle, die zur Verfügung steht und anhand dessen sie ihre Bewertungen eines potenziellen Partners vornehmen und letztlich ihre Kontaktentscheidungen treffen müssen. Wie zuverlässig und vertrauenswürdig sind allerdings die im Online-Profil der Nutzer enthaltenen Informationen über die eigene Person? Wir fragen deshalb zunächst, wie verbreitet unwahre Selbstdarstellungen in Nutzerprofilen auf Online-Kontaktbörsen sind. Unsere Hauptfragestellung lautet dann: Welche Merkmale der Akteure können die beobachtbaren Muster der unwahren Selbstdarstellung im Nutzerprofil erklären? Diese Fragestellung richtet sich auf die Untersuchung des Zusammenhangs der Handlungsvoraussetzungen, mit denen Akteure in den digitalen Partnermarkt einer Kontaktböse eintreten, und den damit verbundenen Handlungen und Strategien der Akteure. Unsere Analyse der unwahren Selbstdarstellung stützt sich auf die in der Partnerwahlforschung diskutierten und für die Paarbildung als zentral charakterisierten Merkmale der Bildung und der physischen Attraktivität (vgl. z.B. Blossfeld/Timm 1997, 2003; Murstein 1972; Stevens et al. 1990; Mulford et al. 1998; Franzen/Hartmann 2001). Gleichzeitig sind dies wahrnehmbare Merkmale, die die Chancen der Menschen auf soziale Wertschätzung beeinflussen und damit wesentlich zur Produktion und Reproduktion sozialer Ungleichheiten beitragen.

Nachfolgend werden wir unsere Fragestellung theoretisch strukturieren und aus diesen Überlegungen Hypothesen zur Erklärung unwahrer Selbstdarstellungen von Männern und Frauen bei der Online-Partnersuche ableiten. Es folgen eine Beschreibung der Datengrundlage, die wir zur Beantwortung unserer Fragestellungen und Prüfung der Hypothesen heranziehen, und die Darstellung der empirischen Befunde, die wir abschließend zusammenfassend diskutieren. 


\section{Theoretische Überlegungen und Hypothesen}

Während die Partnersuche außerhalb des Internets, also in nicht-virtuellen Alltagskontexten, durch die Situation der unmittelbaren Anwesenheit der Akteure gekennzeichnet ist, entfällt bei Online-Kontaktbörsen dieses Moment der unmittelbaren Erfahrung des Gegenübers, was bis zur Unsicherheit hinsichtlich der gesamten Identität des potenziellen Partners führen kann. Auch in Offline-Kontexten stellt die Unsicherheit hinsichtlich einzelner Eigenschaften des Gegenübers ein Problem dar (Goffman 2008), sie erweist sich im virtuellen Kontext jedoch als ungleich virulenter. Denn aufgrund der spezifischen Kontextbedingungen der Partnersuche auf Online-Kontaktbörsen - der Anonymität der Interagierenden, der informationellen Sparsamkeit der Interaktion, der dyadischen Natur der Interaktionsbeziehungen und damit dem Fehlen von Reputations- und Kontrollmechanismen durch Dritte - ist diese Form des Kennenlernens durch ein hohes Maß an Informationsasymmetrie zugunsten des Senders von Informationen gekennzeichnet (vgl. Schmitz 2010; Schulz/Zillmann 2009; Donath 1999). Der Empfänger von Informationen ist wesentlich schlechter über den wahren Zustand wichtiger Eigenschaften in den Nutzerprofilen anderer, wie beispielsweise deren Alter, Bildungsniveau oder Familienstand, informiert als der Sender. Für den Empfänger dieser Informationen ergibt sich daraus ein hohes $\mathrm{Ma} ß$ an Unsicherheit über die tatsächlichen Eigenschaften potenzieller Interaktionspartner. Dadurch werden auf diesem Partnermarkt einerseits unwahre Selbstdarstellungen auf Seiten des Senders von Informationen sehr viel wahrscheinlicher und sind andererseits auf Seiten des Empfängers der Informationen wesentlich schwieriger zu entlarven.

In ihrer eher subtilen und indirekten Form, dem Verschweigen von Informationen, sind unwahre Darstellungen zumindest in den standardisierten Textfeldern durch das Anzeigen von Informationslücken, etwa durch das Einblenden von „keine Angabe“-Antworten, offensichtlich. Treten sie jedoch in Form falscher Informationen auf, wie die Angabe eines falschen Bildungsniveaus oder Alters im Nutzerprofil, sind unwahre Selbstdarstellungen nicht unmittelbar beobachtbar. Oftmals stellen sich derartige Falschinformationen erst mit einem persönlichen Treffen heraus und damit zu einem Zeitpunkt, an dem womöglich bereits viel Zeit und emotionale Bindung in die Interaktionsbeziehung investiert wurde und unter Umständen andere Interaktionsbeziehungen zugunsten dieser nicht fortgeführt wurden. Sie erhöhen damit seitens des Partnersuchenden das Risiko einer verfehlten Investition. Die Etablierung einer Paarbeziehung hängt damit ganz wesentlich davon ab, ob die Akteure das Vertrauensproblem auf dem Online-Partnermarkt lösen und damit Vertrauen in einander aufbauen können, das zu einem späteren Zeitpunkt, z.B. bei einem ersten persönlichen Treffen durch die Offenbarung von (gravierenden) Falschinformationen, nicht wieder zerstört wird.

Diese Ausführungen haben bereits angedeutet, dass Akteure auf diesem Partnermarkt gleichzeitig Sender und Empfänger von Informationen sind und damit die Vertrauensprobleme schaffen, mit denen sie im Perspektivwechsel wieder konfrontiert werden. Diese Untersuchung konzentriert sich auf die Perspektive des Senders von Informationen, also auf die Perspektive des Selbstdarstellers. Dennoch werden wir nachfolgend beide Perspektiven theoretisch berücksichtigen, da sie ineinander greifen und interdependent sind. 
Theoretische Überlegungen zur Selbstdarstellung: Die Perspektive des Senders von Informationen

Mithilfe der Selbstdarstellung werden Informationen über die eigene Person anderen Akteuren übermittelt. Diese Informationen werden vom Gegenüber genutzt, um in Erfahrung zu bringen, wer der andere überhaupt ist. Auf dieser Grundlage werden Bewertungen vorgenommen und Erwartungen hervorgerufen, ob der Kontakt mit dem potenziellen Interaktionspartner als lohnend erscheint. Diese Informationen tragen also - in Goffmans (2008, 1980) Worten - dazu bei, die Situation zu definieren, in der sich Akteure befinden, um darauf aufbauend Handlungsentwürfe zu planen und letztlich Handlungen zu vollziehen. Jedoch ist die Selbstdarstellung nicht nur eine „Serviceleistung“ (Lenz 2006: 153), die Akteure füreinander erbringen, sondern sie erwächst ganz wesentlich aus dem Ziel der Einflussnahme auf das Handeln anderer, vor allem auf deren Handeln gegenüber der eigenen Person (Goffman 2008: 7). Diese Einflussnahme wird dadurch bewirkt, dass Akteure mithilfe einer gezielten Informationsbereitstellung die Definition der Situation ihres Gegenübers - des Empfängers von Informationen - in der Art beeinflussen, dass dieser veranlasst wird, bestimmte Handlungen auszuführen oder zu unterlassen. Im OnlineDating haben Akteure damit die Möglichkeit, mithilfe ihrer Selbstdarstellung im Nutzerprofil ihr Netzwerk an Kontakten - sowohl in quantitativer als auch in qualitativer Hinsicht - zu beeinflussen.

Bei Online-Kontaktbörsen kann man Profilinszenierungen analytisch als Zwischenziel bzw. als „Zwischengut“ (Esser 1999; Ormel et al. 1999) für die Realisierung des individuellen Nutzens in Form langfristiger sozialer Wertschätzung durch potenzielle Partner betrachten. Eine attraktive Darstellung der eigenen Person anhand des Profils ist nur der ,erste Schritt“ auf dem Weg zum letztendlichen Ziel der Etablierung einer Paarbeziehung. Das Profil eines Nutzers bestimmt jedoch in besonderem Maß seine Aufmerksamkeits- und Kontaktchancen, also die Wahrscheinlichkeit, dass dieser in der Masse der konkurrierenden Nutzer wahrgenommen, positiv beurteilt und kontaktiert wird oder sein Kontaktversuch mit einer Antwort bedacht wird. Bei der Selbstinszenierung insbesondere im Nutzerprofil fließen folglich die angenommenen Erwartungen des Gegengeschlechts - die Erwartungserwartungen - ein. Die Erwartungserwartungen werden dadurch zur wesentlichen Grundlage für das inszenatorische Handeln der Akteure. Dies schlägt sich allgemein in der Auswahl der bereitgestellten Informationen über die eigene Person nieder, darüber hinaus aber auch dergestalt, dass die das Gegengeschlecht interessierenden Merkmale betont und gegebenenfalls übertrieben werden. Damit besitzen Selbstdarstellungen häufig eine Tendenz zur Idealisierung, die nicht selten schon als Täuschungen (fabrications) bezeichnet werden können (Goffman 2008, 1980; vgl. auch Lenz 2009). Täuschungen sind intendierte Transformationen der Situationsrahmung des Gegenübers und damit ein Mittel über bewusstes Verbergen oder gar Fälschen von Informationen auf die Situationsdefinition des Gegenübers in gewünschter Weise Einfluss zu nehmen (Goffman 1980: 98).

Theoretische Überlegungen zum Prozess der Partnerwahl: Die Perspektive des Empfängers von Informationen

Die Partnerwahl basiert nicht auf einer singulären Entscheidung, sondern sie kann als Prozess charakterisiert werden (vgl. z.B. Skopek et al. 2009; Hill/Kopp 2006; Murstein 
1970, 1986; Kerckhoff/Davis 1962). Am Anfang des Partnerwahlprozesses steht zunächst die Entscheidung darüber, mit welchen Frauen oder Männern jemand überhaupt näher in Kontakt kommen möchte. Im Online-Dating entsteht dieser allererste Kontakt über das Schreiben einer E-Mail-Nachricht. Wie erfolgt jedoch diese Entscheidung, das heißt wie wählen Akteure aus den zur Verfügung stehenden Personen potenzielle Kontaktpartner aus, aus denen in späteren Phasen des Partnerwahlprozesses möglicherweise feste Paarbeziehungen oder sogar Ehen entstehen?

Ein allgemeines und damit vielseitig anwendbares theoretisches Modell dafür liefert die soziale Austauschtheorie (vgl. z.B. Blau 1964, 1968; Edwards 1969; Huston/Burgess 1979). Soziale Beziehungen werden in diesem Ansatz als Tauschbeziehungen beschrieben. Getauscht werden - symbolisch betrachtet - Ressourcen zwischen Frauen und Männern. Akteure bewerten ihre sozialen Beziehungen dabei hinsichtlich antizipierter Nutzenund Kostenaspekte, also unter Vorwegnahme zukünftiger Erwartungen, die sie an den potenziellen Interaktionspartner richten. Demnach initiieren Akteure dann soziale Beziehungen, wenn sie die Interaktion mit dem potenziellen Partner als belohnend und profitabel einschätzen. Diese Perspektive impliziert die Annahme eines rationalen Akteurs, der sein Verhalten an der Maximierung seines individuellen Nutzens, hier in Form eines möglichst „optimalen“ Partners ausrichtet.

Die Annahme einer optimalen nutzenmaximierenden Partnerwahl scheint jedoch sehr unwahrscheinlich, wenn nicht gar unmöglich (vgl. Blossfeld/Timm 1997, 2003; Skopek et al. 2009; Todd/Miller 1999; Oppenheimer 1988). Deshalb gehen neuere, realistischere Annahmen davon aus, dass Akteure nicht nach einem Optimum, sondern vielmehr - orientiert an Simons Prinzip des „satisficing“ (vgl. Simon 1956, 1990) - nach einer zufriedenstellenden Lösung streben. Demnach suchen Akteure nicht nach dem perfekten Partner, sondern beenden die Suche, sobald ein akzeptabler Partner gefunden wird. Als akzeptabel wird ein potenzieller Partner dann bewertet, wenn er ein bestimmtes Mindestmaß bzw. minimales Anspruchsniveau erfüllt (vgl. Todd/Miller 1999; Blossfeld/Timm 1997, 2003; Oppenheimer 1988). Das Anspruchsniveau dient dabei als subjektive Referenz, an der potenzielle Partner gemessen werden. Damit werden Personen, die eine zu geringe Ressourcenausstattung - und damit einen zu geringen Partnerwert (vgl. Todd/Miller 1999) - signalisieren, als potenzielle Partner abgelehnt. Die Ressourcenausstattung eines Akteurs korrespondiert also mit seinem Partnerwert und bestimmt zugleich seine Gelegenheiten zum sozialen Tausch. Der Partnerwert deutet damit auf die Verhandlungsposition des Akteurs am digitalen Partnermarkt hin, in dem um Aufmerksamkeits- und Kontaktchancen konkurriert wird (vgl. Schmitz/Skopek 2011).

\section{Erweiterung der theoretischen Überlegungen zur Selbstdarstellung}

Mit den vorangestellten Überlegungen zur Partnerwahl und damit einer idealtypischen Perspektive des Empfängers von Informationen können wir die komplementäre Perspektive des Senders bzw. Selbstdarstellers ergänzen und fortführen. An diese Überlegungen anknüpfend ergeben sich für Akteure, die eine vergleichsweise geringe Ressourcenausstattung und damit einen geringen Partnerwert aufweisen, verschiedene idealtypische Handlungsstrategien, um die damit verbundenen Benachteiligungen in Form verringerter Kontakt- und Tauschchancen zu kompensieren. Eine dieser möglichen Handlungsstrategien ist die Kompensation von Nachteilen durch den strategischen Rückgriff auf das Mittel der unwahren Selbstdarstellung. 
Damit ist zu erwarten, dass nur einigen Akteuren der Rückgriff auf Unwahrheiten im Profil oder im Kommunikationsverlauf als hilfreiche Strategie erscheint. Der wahrgenommene Nutzen und damit die Neigung zum strategischen Rückgriff auf Unwahrheiten sollte also mit dem Partnerwert eines Akteurs variieren; Akteure mit geringem Partnerwert sollten eher versucht sein, auf Unwahrheiten bei der Selbstdarstellung im Nutzerprofil zurückzugreifen. Denn Akteure, die sich selbst gute Chancen am Partnermarkt ausrechnen, müssen sich nicht abweichend von ihren Merkmalen darstellen. Diejenigen Akteure, die sich jedoch geringe Chancen auf eine Realisierung ihres Anspruchsniveaus bzw. ihrer Präferenzen hinsichtlich potenzieller Partner ausrechnen, sind womöglich dazu veranlasst, in ihrer Selbstdarstellung auch auf Mittel der Täuschung zurückzugreifen. ${ }^{2} \mathrm{Zu}-$ dem sind Täuschungen alltägliche Handlungen und nicht auf den Kontext des Internets begrenzt. ${ }^{3}$ Täuschungen werden jedoch insbesondere dann auftreten, wenn eine starke Konkurrenz sowie eine hohe Zielkongruenz am Markt herrschen, also viele Akteure um das gleiche Gut wetteifern. Eine Online-Kontaktbörse lässt sich als ein solcher Partnermarkt beschreiben, Hinzu kommt die im Vergleich zu nicht-virtuellen Kontexten des Kennenlernens erhöhte Unsicherheit bezüglich der tatsächlichen Eigenschaften des Interaktionspartners. Diese Rahmenbedingungen von Online-Kontaktbörsen lassen attraktivitätssteigernde Korrekturen der Selbstdarstellung im Vergleich zu den nicht-virtuellen Kontexten des Alltags wahrscheinlicher werden.

Mit dem theoretischen Konzept des Partnerwertes wird die Gesamtattraktivität eines Akteurs beschrieben, die von seiner Ressourcenausstattung abhängt. Implizit darin enthalten ist, dass Akteure ein Bündel verschiedener Ressourcen bzw. Merkmale haben und damit zahlreiche als attraktiv, aber auch als unattraktiv wahrgenommene Merkmale (vgl. Schoen/Wooldredge 1989; Schmitz 2010). Mit diesen Überlegungen können wir nun den allgemein formulierten Mechanismus der Variation unwahrer Selbstdarstellung in Abhängigkeit vom Partnerwert des Akteurs weiter differenzieren.

Aus der Perspektive des sich am Partnermarkt darstellenden Akteurs ist damit naheliegend, dass er versucht, Nachteile in einem bestimmten Merkmal, z.B. im Bildungsniveau, durch Unwahrheit in eben diesem Merkmal auszugleichen. Diesen Ausgleich bezeichnen wir im Folgenden als spezifische Kompensation. Bei der Profildarstellung auf Online-Kontaktbörsen ist diese Strategie aus technischer Sicht leicht umzusetzen und relativ folgenlos, da keine Sanktionen durch Dritte erfolgen können. Allerdings stößt diese Strategie recht bald an Grenzen, denn Akteure können nur im geringen Umfang ein als unattraktiv wahrgenommenes Merkmal in ihrer Profildarstellung beschönigen, wenn sie den potenziellen Partner tatsächlich für eine (wie auch immer geartete) Beziehung gewinnen möchten. Behauptet ein kleinwüchsiger Mann beispielsweise, dass er eine durchschnittliche Körpergröße besitzt, mag das zunächst seine Aufmerksamkeitschancen und

2 Schmitz, Hofmann und Zillmann (2010) konnten in ihrer qualitativen Untersuchung Hinweise darauf finden, dass insbesondere Akteure mit einem niedrigen Partnerwert auf Strategien der Täuschung zurückgreifen, um ihre Chancen, mit potenziellen Partnern überhaupt in Kontakt zu kommen, zu erhöhen. Damit verbunden ist die Hoffnung der Akteure durch das Ausspielen anderer Stärken, z.B. durch besonderen Humor, derartige „Notlügen“ verziehen zu bekommen und den potenziellen Partner letztlich überzeugen zu können.

3 Im Alltag treten Täuschungen überwiegend in Form kleinerer Unwahrheiten und Lügen sowie oft auch in Form so genannter ,white lies“ (Goffman 1984: 69), beispielsweise aus Höflichkeit oder gar zum Schutz anderer, auf (vgl. auch Nyberg 1993; Ekman 1989; Barnes 1994). 
damit seine Kontaktchancen in Form vermehrter Zuschriften und Antworten steigern, seine Tauschchancen im Sinne einer sich verstetigenden Beziehung erhöht er dadurch aber nicht. Im Gegenteil: diejenigen Frauen, die er für ein persönliches Treffen gewinnen kann, werden nach einer ersten Begegnung höchstwahrscheinlich enttäuscht sein und auf weitere Treffen verzichten. Und so zeigt sich auch tatsächlich, dass sich Akteure auf Kontaktbörsen zwar der Strategie der spezifischen Kompensation bedienen, sie tun dies jedoch meist nur in Form einer graduellen Optimierung. Männer machen sich beispielsweise nur etwas größer und Frauen nur etwas schlanker (vgl. Hancock et al. 2007; Toma et al. 2008). Sie handeln dabei entlang gesellschaftlich geteilter Konventionen, und zwar einerseits hinsichtlich einer optimalen Merkmalsausprägung. Diese Konventionen geben Akteuren in Form von Leitbildern und Geschlechterrollen Orientierung im Hinblick darauf, was überhaupt am Partnermarkt als attraktive Eigenschaft bei einem Mann und einer Frau gilt. Andererseits gelten diese Konventionen auch hinsichtlich der erwarteten und als normal geltenden Qualität und Quantität der Unwahrheiten. Nicht jede Täuschung oder das was umgangssprachlich auch als Lüge bezeichnet wird, wird gleichermaßen stark moralisch verurteilt. Es ist anzunehmen, dass Akteure diese Konventionen, die als ungeschriebene Regeln am Partnermarkt gelten, bei der Anwendung ihrer Strategien berücksichtigen. Einige Akteure werden also versuchen, ihre restringierte Position am Partnermarkt zu kompensieren, indem sie ihre wesentlichen Restriktionen auf eine konventional zulässige Weise in ihrer Selbstdarstellung beschönigen.

Die Strategie der spezifischen Kompensation ist aber nicht die einzig denkbare Logik des Umgangs mit vermeintlichen oder tatsächlichen Nachteilen. Die konventionalen Grenzen der Anwendung der spezifischen Kompensation lassen weitere Kompensationsstrategien erwarten. So ist aus Sicht eines Akteurs denkbar, dass Nachteile in einem Merkmal auch durch Stärken in anderen Merkmalen ausgeglichen werden können. Beispielsweise könnte ein Akteur seine geringe Ausstattung mit Bildungsressourcen auch durch besondere physische Attraktivität kompensieren. Ein anderes Beispiel, das ebenfalls dieser Logik folgt, stellt einen Akteur dar, der seine tatsächliche oder vermeintliche physische Unattraktivität zusätzlich zur konventional zulässigen Beschönigung dieses Merkmals dadurch zu kompensieren versucht, indem er - den Tatsachen nicht entsprechend - einen hohen Bildungsstatus vorgibt. Diese Strategie werden wir im Folgenden als unspezifische Kompensation bezeichnen. Akteure greifen also auf die den Konventionen entsprechenden Möglichkeiten des Zwischenziels einer optimierten Profildarstellung zurück, die nicht zwingend eine direkte Kompensation ihrer Merkmalsschwächen zum Gegenstand haben müssen. Vielmehr kann ein restringierter Partnerwert auch unwahre Selbstdarstellungen bei Merkmalen begünstigen, die nicht oder nur im geringen $\mathrm{Ma}$ für ihre schlechten Chancen ursächlich verantwortlich sind. Diese Überlegung leitet sich aus der Vorstellung ab, dass Akteure aus einem Bündel von attraktiven aber auch weniger begehrten Merkmalen bestehen. Ein strategisch reflektierender Akteur nimmt seine Tauschchancen vor dem Hintergrund seiner Position am Partnermarkt vorweg und versucht, seinen Gesamteindruck zu verbessern, um zunächst in der Masse möglicher Kontakte überhaupt erst wahrgenommen zu werden und schließlich eine Interaktion mit einem potenziellen Partner wahrscheinlicher zu machen. Dabei müssen Akteure nicht unbedingt anstreben, sich als optimal im Sinne der bestmöglichen Merkmalsausprägung darzustellen, sondern vielmehr als normal im Sinne einer nicht unterdurchschnittlichen Merkmalsaus- 
prägung. ${ }^{4}$ Bei der Gestaltung des eigenen Profils kommt es also auch darauf an, seine Kontaktchancen durch ein Vermeiden der Zurschaustellung von als negativ angenommenen Merkmalen zu erhöhen, um von potenziellen Partnern aufgrund ihrer erwarteten Kriterien bei der Partnersuche und -selektion nicht einfach ausgeschlossen zu werden. Insbesondere auf einem Partnermarkt, der wie das Online-Dating durch eine Überfülle möglicher Partner charakterisiert ist, suchen Menschen vermehrt nach bestimmten Eigenschaften und vermeiden andere. ${ }^{5}$

Mit der spezifischen und unspezifischen Kompensation haben wir zwei idealtypische Strategien aufgezeigt, mit denen Akteure eine geringe Ressourcenausstattung zu kompensieren versuchen. Wir werden diese Überlegungen im Folgenden unter Berücksichtigung einer Geschlechterperspektive in Bezug auf die für unsere Analyse relevanten Merkmale Bildung und physische Attraktivität konkretisieren. Gleichzeitig liefert diese Diskussion die Grundlage zur Spezifikation der geschlechts- und ressourcenspezifischen Hypothesen.

\section{Die Erwartung gegengeschlechtlicher Partnerpräferenzen}

In den bisherigen Ausführungen haben wir zwei Akteure unterschieden, die die Täuschung konstituieren: der Akteur, der täuscht, also der Sender falscher und selektiver Informationen, und der Akteur, der getäuscht wird, also der Empfänger dieser falschen und selektiven Informationen. In diesem dyadischen Verhältnis müssen jedoch noch weitere Akteure unterschieden werden, die mittelbar im Täuschungsakt involviert sind. Analytisch muss nämlich das Modell, an dem sich der Täuschende orientiert (vgl. Gambetta 2005: 234) ebenso berücksichtigt werden wie Dritte, die die Täuschung sanktionieren könnten. Da das Sanktionierungspotenzial Dritter auf Online-Kontaktbörsen äußerst gering ist, stellt sich insbesondere die Frage, an welchem Modell sich Akteure bei der Darstellung ihrer Person orientieren. Zur Beantwortung dieser Frage ist Meads Konzept des signifikanten und generalisierten Anderen aufschlussreich (Mead 1934). Mit diesem Konzept ist die Fähigkeit des Akteurs zur Perspektivübernahme angesprochen, um damit die Reaktion des Gegenübers zu antizipieren und letztlich auch den Verlauf einer (potenziellen) Interaktion einzuschätzen. Im Online-Dating lässt sich eine Unterscheidung danach treffen, ob im Rahmen des Nutzerprofils ein generalisierter Anderer getäuscht wird oder im Rahmen einer dyadischen Interaktion ein signifikanter bzw. konkreter Anderer. Mithilfe der Selbstdarstellung über das Nutzerprofil präsentiert sich ein Nutzer auf dem digitalen Partnermarkt jedoch in jedem Fall gegenüber einem generalisierten Anderen in Vorwegnahme der Erwartungen potenzieller (Kontakt-)Partner. Diese generalisierte Kommunikation ist für Akteure die erste Möglichkeit, sich für die Partnersuche ins rechte Licht zu rücken.

Die Vorwegnahme der Erwartungen eines generalisierten Anderen verweist auf die in einer Gesellschaft etablierten Geschlechterrollen und -leitbilder im Kontext privater Bezie-

4 Und so schreibt z. B. Goffman: „Wegen der großen Belohnungen, die die Tatsache, als normal betrachtet zu werden, mit sich bringt, werden fast alle Personen, die die Möglichkeit haben, zu täuschen, dies auch bei irgendeiner Gelegenheit absichtlich tun."(Goffman 1975: 96).

5 Lenton und Stewart (2008) konnten zeigen, dass mit zunehmendem Umfang des Angebots potenzieller Partner die Auswahlstrategien der Akteure zunehmend Eigenschaften im Unterschied zu Alternativen in Form ,ganzheitlicher" Akteure zum Gegenstand hatten und dabei zunehmend weniger Kompromisse bei der Auswahl potenzieller Partner eingegangen wurden. 
hungen, an denen sich Akteure bei ihrer Selbstdarstellung orientieren. Es sind dies insbesondere die jeweiligen Erwartungen im Hinblick auf die Partnerpräferenzen des Gegengeschlechts. Vor diesem Hintergrund stellt sich die Frage, welche partnerschaftlichen Präferenzen Männer und Frauen heute im Allgemeinen (in unserer Gesellschaft) haben und welche Merkmale somit Frauen und Männer als attraktiv und begehrenswert erscheinen lassen. ${ }^{6}$

Ausgehend von unterschiedlichen Sozialisationsbedingungen, die Frauen und Männer erfahren haben sowie den sich daraus bildenden unterschiedlichen Rollenerwartungen, denen Frauen und Männer ausgesetzt sind, lässt sich eine geschlechtsspezifische Strukturierung von Partnerpräferenzen erwarten (vgl. Blossfeld/Drobnič 2001; Skopek 2011). ${ }^{7}$ Beispielsweise zeigte sich auf der Grundlage von Kontaktanzeigenstudien, dass Frauen im Vergleich zu Männern in ihren Angaben häufiger nach Partnern suchen, die finanzielle Sicherheit (Butler-Smith et al. 1998; Hirschman 1987; Harrison/Saeed 1977) und „gute“ statusrelevante Merkmale wie höhere Bildung oder berufliche Position (Hassebrauck 1990; Kaupp 1968) bieten und darüber hinaus älter sind als sie selbst (Harrison/Saeed 1977). Demgegenüber zeigten Männer eine deutliche Präferenz für jüngere Frauen (Campos et al. 2002; Cameron et al. 1977) und physisch attraktive Partnerinnen (z.B. Hirschman 1987). Männer legen nach diesen Studien bei der Auswahl von potenziellen Partnerinnen vor allem Wert auf Jugendlichkeit und Schönheit. Weitere Studien zu Partnerpräferenzen und Geschlechterstereotypen zeigen, dass bei Männern auch die Körpergröße ein relevantes Merkmal zu sein scheint (vgl. z.B. Skopek 2011; Pawloswki/Koziel 2002; Biernat et al. 1991; Shepperd/Strathman 1989; Lynn/Shurgot 1984; Gillis/Avis 1980).

Damit stellen der männliche Status und die männliche Körpergröße zwei erhärtete Kriterien dar, die über Erfolg und Misserfolg am Partnermarkt entscheiden können. Es ist deshalb anzunehmen, dass sich Frauen dementsprechend - angesichts der Fülle potenzieller Partner auf Kontaktbörsen - im ersten Schritt auf Profile beschränken, die ihren Präferenzen hinsichtlich dieser beiden wichtigen Merkmale entsprechen. Indem Männer bei der Partnersuche diese partnerschaftlichen Präferenzen von Frauen vorwegnehmen, sollten sie, wenn sie sich des strategischen Mittels der Täuschung bedienen, also vor allem in den Merkmalen Bildung und Körpergröße einen Ansatzpunkt zur Optimierung ihrer Präsentation sehen. Frauen hingegen sollten bei ihrer Profildarstellung vor allem auf Merkmale der physischen Erscheinung Wert legen, wie sie in den Angaben zum Körpergewicht (insbesondere relativ zur Körpergröße als Body Mass Index) sowie in den zur Verfügung gestellten Fotographien über die eigene Person zum Ausdruck kommt. ${ }^{8}$

Diese präferenzbasierte Argumentation, die der Perspektive des individuellen Akteurs entspricht, ist allerdings nur eine Seite der Medaille. Abstrahiert man von der individuel-

6 Es geht hier also um die Frage nach absoluten Attraktivitätsbewertungen bzw. Partnerpräferenzen. Davon abzugrenzen sind relationale Attraktivitätsbewertungen, bei der die Attraktivität eines Merkmals des Gegenübers relativ zur eigenen Merkmalsausprägung bewertet wird.

7 Eine grundlegend andere Erklärung geschlechtsspezifisch strukturierter Partnerpräferenzen liefern evolutionsbiologische Ansätze (vgl. dazu bspw. die Studien von Buss und Barnes 1986; Buss und Schmitt 1993; siehe auch die Diskussion soziologischer und evolutionsbiologischer Erklärungsansätze von Geschlechterunterschieden in partnerschaftlichen Präferenzen in dem Artikel von Eagly und Wood 1999).

8 Auch das Alter der Frauen ist ein Signal für ihre physische Attraktivität (vgl. z.B. England/McClintock 2009). Zur Bedeutung des Alters für die Attraktivität von Frauen und Männern siehe auch den Beitrag von Skopek et al. in diesem Band. 
len Perspektive und wechselt zu einer übergeordneten Perspektive - der Perspektive des Partnermarktes - gerät zusätzlich in den Blick, dass die Attraktivität eines Merkmals zwar durch allgemein geteilte Vorstellungen darüber, was als attraktiv gilt, bestimmt wird. Sie wird darüber hinaus aber auch wesentlich durch die Verfügbarkeit dieses Merkmals am Partnermarkt bestimmt. Damit hängt der Wert einer bestimmten Eigenschaft, wie z.B. eine schlanke Figur, auch davon ab, wie groß das Angebot dieser Eigenschaft am Partnermarkt ist und inwieweit sie damit Gegenstand von Konkurrenz ist (vgl. z.B. Stauder 2008; Klein/Stauder 2008). Die Attraktivität eines Merkmals wird damit also vom Zusammenspiel individueller Vorlieben für eben dieses Merkmal und von Marktprozessen und damit den Gelegenheiten am Partnermarkt bestimmt.

\section{Methodisches Vorgehen}

\section{Datengrundlage und Analysestichprobe}

Für die Beantwortung der Fragestellungen und die Prüfung der Hypothesen greifen wir auf Daten zurück, die im Rahmen einer Online-Befragung auf einer großen deutschen Online-Kontaktbörse erhoben wurden. ${ }^{9}$ Die Datenerhebung auf der Plattform erfolgte vom 22. Juni 2009 bis 15 . April 2010. Alle angemeldeten und aktiven ${ }^{10}$ Nutzer der Plattform wurden per E-Mail-Nachricht zur Teilnahme an der Befragung eingeladen. Insgesamt nahmen 3.535 Online-Dater an der Befragung teil. Das entspricht einer Ausschöpfungsquote von 10 Prozent. ${ }^{11}$

Für die Prüfung der Hypothesen haben wir eine Analysestichprobe konstruiert. Da das Interesse darin besteht, Unwahrheiten in der Selbstdarstellung von Frauen und Männern im Prozess der Partnersuche zu analysieren, umfasst die Analysestichprobe nur Befragte, die explizit angeben, auf Partnersuche zu sein; das sind 63 Prozent der Befragten. Damit wurden Personen ausgeschlossen, die auf die Frage, ob sie derzeit auf der Suche nach einem Partner sind, mit „nein“ oder „weiß nicht“ antworteten. Weiterhin enthält die Analysestichprobe nur heterosexuelle Partnersuchende, also Frauen, die ausschließlich nach Männern suchen und umgekehrt. Schließlich haben wir das Alter auf 18 bis 80 Jahre beschränkt. Befragte, die einen fehlenden Wert bei der Altersangabe enthielten, wurden beibehalten. Insgesamt reduziert sich damit die Fallzahl von ursprünglich 3.535 auf 2.113 Fälle. In unserer Analysestichprobe sind 1.350 Männer (64 Prozent) und 763 Frauen (36 Prozent) enthalten. Das Durchschnittsalter der Nutzer beträgt 41 Jahre. Eine detaillierte Beschreibung der Analysestichprobe enthält Tabelle A.1 im Anhang.

9 Wir danken der Deutschen Forschungsgemeinschaft (DFG) für die finanzielle Förderung des Projektes „Prozesse der Partnerwahl auf Online-Kontaktbörsen“, in dessen Rahmen wir die Datenerhebung durchgeführt haben. Insbesondere danken wir unserem Kooperationspartner, der die Datenerhebung auf seiner Online-Plattform erst ermöglicht und unterstützt hat.

10 Ein Nutzer galt dann als aktiver Nutzer der Plattform, wenn er sich in den letzten sechs Monaten (Referenzzeitpunkt ist der Beginn unserer Befragung) mindestens einmal auf der Plattform eingeloggt hat. Inaktive Nutzer erhielten keine Einladung; insgesamt wurden 35.235 Nutzer zur Befragung eingeladen.

11 Eine Auflistung der Ausschöpfungsquoten nach unterschiedlich definierten Subgruppen ist bei Skopek (2010) zu finden. 
Operationalisierung der abhängigen und unabhängigen Variablen

In der Online-Befragung wurden Nutzer zu ihren subjektiven Wahrnehmungen und Interpretationen, nach individuellen Lebenslaufereignissen, zu Bedingungen außerhalb der Online-Kontaktbörse sowie zur unwahren Selbstdarstellung befragt. Die Frage nach der eigenen unwahren Selbstdarstellung im Nutzerprofil war wie folgt formuliert: „Manche Nutzer möchten ihre Chancen verbessern, indem sie in ihren Profilangaben etwas mogeln. Stellst du dich in deinem Profil auch manchmal anders dar, als du eigentlich bist bezüglich ...".12 Danach wurden verschiedene Angaben, die Nutzer in ihrem Profil über sich preisgeben können, aufgelistet wie beispielsweise Alter, Größe, Gewicht, Kinderzahl und Bildung. Die Befragten konnten jede dieser Angaben mit ,ja“ oder „nein“ beantworten. Unsere abhängigen Variablen für die nachfolgenden Analysen sind die (selbstberichteten) unwahren Auskünfte über die eigene Person im Nutzerprofil im Bildungsniveau sowie in den Größen- und Gewichtsangaben. Die Größen- und Gewichtsangaben verwenden wir als Indikator für die physische Attraktivität der Nutzer. Zum einen folgen wir damit anderen Untersuchungen (vgl. z.B. Franzen/Hartmann 2001; Lynn/Shurgot 1984; Skopek 2011; Tovée et al. 1998, 1999, 2002). Zum anderen sind die Nutzerprofile im Vergleich zu den zur Verfügung stehenden Informationen im nicht-virtuellen Alltag wesentlich weniger reichhaltig. Es ist anzunehmen, dass dann aber gerade diese wenigen Angaben, die zur Verfügung stehen, beispielsweise zur physischen Attraktivität des Gegenübers - wie sie in Größen- und Gewichtsangaben neben eventuell zur Verfügung gestellten Fotografien zum Ausdruck kommen - zur Beurteilung des Gegenübers sehr wichtig werden.

Für die Analysen der unwahren Selbstdarstellung verwenden wir Selbstauskünfte der Befragten. Es ist generell schwierig, Täuschungen in sozialen Interaktionen empirisch zu erfassen (vgl. z.B. Hettlage 2003; Lenz 2003; Schmid 2003). Besonders schwierig zu erfassen sind dabei Täuschungen, auf die Akteure selbst zurückgreifen wie beispielsweise eine unwahre Darstellung der eigenen Person gegenüber Anderen. In Bezug auf die hier verwendeten Befragungsdaten ist mit dem Problem sozialer Erwünschtheit zu rechnen (vgl. z.B. Diekmann 2008: 447ff.). Insofern ist anzunehmen, dass der Umfang unwahrer Selbstdarstellung der Akteure auf dem Online-Partnermarkt unterschätzt wird. Neben dieser Einschränkung haben die für unsere Analyse verwendeten Befragungsdaten aber einen entscheidenden Vorteil: Befragungsdaten vermögen es, die Intention der Akteure abzubilden. Denn erst das intentionale und damit bewusste Senden falscher und selektiver Informationen trennt die Täuschung vom Irrtum (Goffman 1980: 98; Simmel 1992, orig. 1908: $388){ }^{13}$

12 Wir haben die Nutzer in der Befragung mit „du“ anstelle von „Sie“ angesprochen und uns damit dem Sprachgebrauch auf der Plattform angepasst.

13 „Nicht, dass der Belogene über die Sache eine falsche Vorstellung hat, erschöpft das spezifische Wesen der Lüge - das teilt sie mit dem einfachen Irrtum; sondern daß er über die innere Meinung der lügenden Person in Täuschung erhalten wird.“ (Simmel 1992, orig. 1908: 388). Damit geht es bei der Täuschung nicht einfach um die Diskrepanz zwischen Darstellung und Wirklichkeit. Die objektive Wahrheit ist damit nicht entscheidend; zum Maßstab wird vielmehr die subjektive Unwahrheit (Hettlage 2003: 12; Schmid 2003: 53; Lenz 2003: 66). Wenn ein Akteur beispielsweise eine falsche Angabe zu seiner Körpergröße macht, dann wird diese Falschangabe erst dann zur Täuschung, wenn er sich dessen bewusst ist und damit absichtlich bei Anderen einen falschen Eindruck erwecken möchte. Ist sich der Akteur über die Abweichung nicht bewusst, kann nur von einem Irrtum gesprochen werden. 
Als unabhängige Variablen für unsere Analysen verwenden wir das erfragte Bildungsniveau, die Körpergröße, das Körpergewicht, das Alter und den Beziehungswunsch des Nutzers. Diese Variablen wurden wie folgt gebildet:

Bildungsniveau. Zur Operationalisierung der Bildungsressourcen des Nutzers verwenden wir in Anlehnung an Blossfeld und Timm (1997, 2003) drei Abstufungen, die aus den Angaben der Befragten zu ihrem Bildungsabschluss und Ausbildungsabschluss zusammenfasst wurden. Wir erhalten damit die folgende hierarchische Abstufung: In der Kategorie „niedriges Bildungsniveau“ sind (1) Personen ohne Schulabschluss, (2) Personen mit Hauptschulabschluss ohne berufliche Ausbildung und (3) Personen mit mittlerer Reife ohne berufliche Ausbildung enthalten. In der Kategorie „mittleres Bildungsniveau“ sind (1) Personen mit einem Hauptschulabschluss in Kombination mit einem beruflichem Ausbildungsabschluss, (2) Personen mit mittlerer Reife und einem beruflichem Ausbildungsabschluss, (3) Personen mit Abitur ohne beruflichen Ausbildungsabschluss und (4) Personen mit Abitur und beruflichem Ausbildungsabschluss enthalten. Schließlich sind in der Kategorie „hohes Bildungsniveau“ Personen enthalten, die (1) einen Fachhochschulabschluss, (2) einen Hochschulabschluss oder (3) eine Promotion als formalen Bildungstitel besitzen. Mithilfe dieser Bildungsklassifikation können die damit verbundenen individuellen Arbeitsmarkt-, Einkommens- und Karrierechancen adäquat abgebildet werden (vgl. Blossfeld/Timm 1997: 443).

Körpergröße und -gewicht. Da uns keine Informationen über die im Nutzerprofil zur Verfügung gestellten Fotografien vorliegen, verwenden wir zur Operationalisierung der physischen Attraktivität die Angaben der Befragten zu ihrer Körpergröße (in Zentimetern) und ihrem Körpergewicht (in Kilogramm). Im Modell zur Erklärung der unwahren Selbstdarstellung des Bildungsniveaus verwenden wir die Größen- und Gewichtsangaben in Relation zueinander in Form des Body Mass Index (BMI). Die Körpergröße nehmen wir in diesem Modell als zusätzliche Variable auf, da die bisherige Forschung gezeigt hat, dass sie ein vom BMI unabhängiges und relevantes Merkmal für die Partnerwahlentscheidung von Frauen zu sein scheint (vgl. z.B. Skopek 2011; Shepperd/Strathman 1989; Gillis/Avis 1980). In den Modellen zur Erklärung der unwahren Selbstdarstellung der Größe und des Gewichts werden Größen- und Gewichtsangaben einzeln in die Modelle aufgenommen.

Alter. Das Alter der Nutzer (in Jahren) wird aus den Angaben zu Geburtsmonat und -jahr sowie dem Befragungszeitpunkt berechnet.

Beziehungswunsch. Als zusätzliche, ressourcenunabhängige Variable verwenden wir in unseren Analysen die Angabe des Befragten, inwiefern er auf der Suche nach einer festen Beziehung ist. Diese Angabe konnte auf einer fünfstufigen Skala von 1 ,trifft überhaupt nicht zu“ bis 5 ,trifft voll und ganz zu“ beantwortet werden. ${ }^{14}$ Diese Variable fügen wir

14 In unseren Analysen verwenden wir diese Angabe als 0/1-kodierte Variable. Der Wert 1 (gebildet aus den Werten 4 und 5 der ursprünglichen Skala) zeigt an, dass der Befragte auf der Suche nach einer festen Beziehung ist; der Wert 0 (gebildet aus den Werten 1 bis 3 der ursprünglichen Skala) entsprechend, dass der Befragte tendenziell nicht auf der Suche nach einer festen Beziehung ist. 
aufgrund unserer theoretischen Überlegungen ein. Darin haben wir argumentiert, dass die Absicht, einen potenziellen Partner auch persönlich zu treffen mit dem letztlichen Ziel der Etablierung einer längerfristigen Beziehung, wesentliche Auswirkungen darauf haben kann, inwiefern auf Unwahrheiten im Nutzerprofil zurückgegriffen wird.

\section{Ergebnisse}

Deskriptive Ergebnisse zur Verbreitung unwahrer Selbstdarstellung im Nutzerprofil Unsere erste Fragestellung betrifft die Verbreitung unwahrer Selbstdarstellung im Nutzerprofil auf Online-Kontaktbörsen. Tabelle 1 zeigt die Angaben der Befragten zur unwahren Selbstdarstellung im eigenen Nutzerprofil entlang zentraler Merkmale.

Tabelle 1: Unwahre Selbstdarstellung im eigenen Nutzerprofil (in Prozent)

\begin{tabular}{|c|c|c|c|c|c|c|}
\hline Unwahre Selbstdarstellung & Gesamt & Männer & Frauen & $\mathrm{N}_{1}$ & $\mathrm{~N}_{2}$ & Phi \\
\hline Irgendeines Merkmals & 26,9 & 27,3 & 26,1 & 538 & 2.002 & - \\
\hline Größe & 6,5 & 7,8 & 4,3 & 130 & $1.997^{\star \star}$ & 0,068 \\
\hline Alter & 10,3 & 11,8 & 7,6 & 205 & $1.997^{\star \star}$ & 0,065 \\
\hline Gewicht & 15,0 & 14,2 & 16,3 & 299 & 2.000 & - \\
\hline Gesuchte Beziehung & 8,3 & 9,9 & 5,5 & 165 & $1.994^{* * *}$ & 0,076 \\
\hline Familienstand & 6,7 & 7,7 & 4,8 & 133 & $1.997^{*}$ & 0,055 \\
\hline Kinderzahl & 5,0 & 5,7 & 3,7 & 100 & $1.993^{*}$ & 0,044 \\
\hline Bildung & 6,2 & 7,1 & 4,7 & 124 & $1.992^{*}$ & 0,047 \\
\hline Foto & 7,3 & 8,1 & 6,0 & 146 & $1.991+$ & 0,039 \\
\hline Geschlecht & 5,2 & 6,0 & 3,9 & 104 & $1.991^{*}$ & 0,045 \\
\hline Anderes Merkmal & 5,5 & 6,7 & 3,5 & 110 & $1.989 * \star$ & 0,068 \\
\hline
\end{tabular}

Signifikanz: ${ }^{+} 0,1 ; * \mathrm{p} \leq 0,05 ; * * \mathrm{p} \leq 0,01 ; * * * \mathrm{p} \leq 0,001$. Quelle: Online-Befragung von Nutzern einer großen deutschen Online-Kontaktbörse; $\mathrm{N}=2.113$; eigene Berechnungen. Anmerkung: $\mathrm{N}_{1}$ gibt die Anzahl der Nutzer wieder, die auf die Frage nach der eigenen unwahren Selbstdarstellung im Nutzerprofil in der ausgewählten Merkmalsdimension mit ,ja“ antworteten. $\mathrm{N}_{2}$ gibt die Anzahl der Nutzer wieder, die die Frage überhaupt (mit ,ja“ oder ,nein“) beantwortet haben.

Insgesamt gibt die Mehrheit der Befragten an, in ihrem Nutzerprofil ehrliche Angaben über die eigene Person zu machen. Lediglich ein Viertel (27 Prozent) der Nutzer gibt an, sich in einem oder mehreren Merkmalen schon unwahr dargestellt zu haben. Zwar ist davon auszugehen, dass einige Nutzer aufgrund der wahrgenommenen sozialen Erwünschtheit nicht zugaben, auf Unwahrheiten zurückgegriffen zu haben. Dennoch kann unsere deskriptive Analyse das Mindestmaß an strategischer Täuschung quantifizieren. Auf Grundlage der selbstberichteten Auskünfte zu Unwahrheiten im Nutzerprofil scheint das eingangs angesprochene Vertrauensproblem zwischen Sender und Empfänger von Informationen kein beträchtliches Ausmaß zu haben. Insofern die Teilnahme an der Befragung und deren Beantwortung nicht selektiv sind, können wir zudem Aussagen über die Struktur der eingeräumten Unwahrheiten treffen.

Hinsichtlich des Bildungsniveaus werden vergleichsweise selten unwahre Angaben gemacht. Es sind jedoch mehr Männer (7 Prozent) als Frauen (5 Prozent), die angeben, in ihrem Bildungsniveau schon falsche Angaben im Nutzerprofil bereitgestellt zu haben. Dieser Unterschied ist statistisch signifikant, was die in unseren theoretischen Überlegun- 
gen diskutierte Relevanz des Bildungsniveaus als Statusressource insbesondere für Männer bestätigt. In den Größenangaben sind es ebenfalls mehr Männer als Frauen, die im Nutzerprofil auf Unwahrheiten zurückgreifen. Auch diesen Zusammenhang haben wir in unseren theoretischen Überlegungen vermutet. Bei den Angaben zum Gewicht wird - relativ zu den anderen Merkmalen - recht häufig die Unwahrheit angegeben. Hier sind es mit 16 Prozent mehr Frauen als Männer (14 Prozent), die diesbezüglich in ihrem Nutzerprofil schon einmal nicht ehrlich waren. Dieser Zusammenhang unterstützt unsere Annahmen, allerdings ist er in unserer Analysestichprobe statistisch nicht signifikant. Die vergleichsweise hohe Anzahl an ,positiven“ Antworten in den Gewichts- und Altersangaben (mit 15 bzw. 10 Prozent) könnte darauf hindeuten, dass Unwahrheiten in diesen Angaben, als vergleichsweise legitim wahrgenommen werden (vgl. Toma et al. 2008: 1031). Zum anderen könnte das auch darauf hindeuten, das Unwahrheiten in „metrischen Variablen" wie den Alters-, Gewichts- und Größenangaben durch die Möglichkeit einer sehr feinen Abstufung leichter umzusetzen sind als Falschangaben in kategorialen Merkmalen und dadurch von einigen Akteuren als sehr erfolgsversprechend wahrgenommen werden. Schließlich sind sie in einem späteren persönlichen Treffen recht schwierig zu entlarven. $\mathrm{Ob}$ jemand beispielsweise fünf Kilogramm weniger angegeben hat als er tatsächlich wiegt, dürfte auch in einer persönlichen Begegnung nur schwer feststellbar sein. Wenn aber die Wahrscheinlichkeit der Entdeckung der Unwahrheit sinkt, sollte ein rationaler Akteur den Nutzen der Unwahrheit höher einstufen; damit steigt gleichzeitig der Erwartungswert der entsprechenden Lüge.

Generell fällt auf, dass sich Männer in allen Merkmalsdimensionen mit Ausnahme des Gewichts häufiger unwahr darstellen als Frauen. Gerade Männer sind aber auf Online-Kontaktbörsen in einer wesentlich verschärfteren Konkurrenzsituation als Frauen, ganz einfach deshalb, weil üblicherweise immer noch mehr Männer als Frauen OnlineKontaktbörsen nutzen (vgl. z.B. Brym/Lenton 2001; Schulz et al. 2008; Sautter et al. 2010). In unserer Analysestichprobe kommen auf jede Frau 1,8 Männer. Deshalb müssen insbesondere Männer auf die Gestaltung ihres Profils Wert legen und unter Umständen auch auf Unwahrheiten zurückgreifen, um ihre Aufmerksamkeits- und damit Kontaktchancen zu steigern und nicht in der Masse potenzieller Partner unterzugehen. Bei den Frauen hingegen entschärft sich die Konkurrenzsituation. Damit ist es für Frauen eher möglich auf den Rückgriff auf Unwahrheiten im Nutzerprofil, aber auch in der späteren Kommunikation, zu verzichten. Wenn es nur wenige Frauen auf diesem Partnermarkt gibt, dann werden auch vermeintlich weniger attraktive Frauen zu begehrenswerten Alternativen, und zwar nur deshalb, weil sie rar sind. Es bestätigt sich aber die erwartete Struktur der eingeräumten Unwahrheiten in der Profildarstellung bei den befragten Frauen. Frauen greifen bei Unwahrheiten eher auf solche Merkmale zurück, die mit Jugend und körperlicher Attraktivität verbunden sind.

\section{Multivariate Analyse der unwahren Selbstdarstellung im Nutzerprofil}

Die deskriptiven Analysen haben gezeigt, dass nur ein relativ geringer Anteil der Befragten berichtet, sich im Nutzerprofil schon unwahr dargestellt zu haben. Zwar liegt uns damit nur eine geringe Anzahl an „,positiven“ Antworten vor. Für unsere statistischen Analysen ist diese Anzahl allerdings ausreichend. In den nachfolgenden multivariaten Analysen tragen wir dieser geringen Anzahl an ,positiven“ Antworten dadurch Rechnung, dass wir die kom- 
plementäre Log-log-Regression verwenden. Dieses Verfahren eignet sich insbesondere bei sehr schiefen Verteilungen einer dichotomen abhängigen Variablen. Eine Formalisierung des Modells ist zum Beispiel bei Agresti (2002: 248ff.), Powers und Xie (2000: 83f.) und Long (1997: 50ff.) zu finden. Mit diesem Verfahren schätzen wir die Wahrscheinlichkeit, dass ein Nutzer bei einer gegebenen Merkmalsausprägung auf Unwahrheiten in der Darstellung seines Bildungsniveaus, seiner Körpergröße oder seines Körpergewichts zurückgreift. In den nachfolgenden Tabellen weisen wir die unstandardisierten Regressionskoeffizienten aus. ${ }^{15}$ Zur Testung der Hypothesen legen wir aufgrund der kleineren Fallzahlen und zur Verringerung des $\beta$-Fehlers ein Signifikanzniveau von 10 Prozent an.

Geschlechts- und ressourcenspezifische Erklärung von Profiltäuschungen in der Bildung Unsere Hauptfragestellung lautete, wie die beobachtbaren Muster der unwahren Selbstdarstellung im Nutzerprofil erklärt werden können. Dazu haben wir ausgehend von den theoretischen Überlegungen zur geschlechtsspezifischen Ressourcenausstattung der Akteure prüfbare Hypothesen zur unwahren Selbstdarstellung abgeleitet. Aufgrund der geschlechtsspezifisch formulierten Hypothesen sind die nachfolgenden Modelle zur Erklärung der unwahren Selbstdarstellung in den Merkmalsdimensionen Bildung, Gewicht und Körpergröße getrennt nach Geschlecht dargestellt. Tabelle 2 zeigt die Ergebnisse zur unwahren Selbstdarstellung des Bildungsniveaus im Nutzerprofil von Männern und Frauen.

Die Kompensation von vermeintlichen oder tatsächlichen Nachteilen in einem Merkmal durch den Rückgriff auf Unwahrheiten in eben diesem Merkmal, die wir als spezifische Kompensation bezeichnet haben, testen wir im ersten Schritt. Modell 1 in Tabelle 1 zeigt die Regressionskoeffizienten des kategorisierten Bildungsniveaus. Für Männer zeigt sich ein monotoner Effekt: mit steigendem Bildungsniveau sinkt die Wahrscheinlichkeit, dass diese ihr Bildungsniveau im Nutzerprofil unwahr darstellen. Männer mit niedrigem Bildungsniveau haben im Vergleich zu Männern mit mittlerem und hohem Bildungsniveau die höchste Wahrscheinlichkeit, hinsichtlich ihres Bildungsniveaus unehrliche Angaben zu machen. Bei Frauen hingegen zeigt sich ein u-förmiger Bildungseffekt. Sowohl Frauen mit niedrigem als auch mit hohem Bildungsniveau haben im Vergleich zu Frauen mit mittlerem Bildungsniveau eine höhere Wahrscheinlichkeit, ihr Bildungsniveau im Nutzerprofil abweichend von den Tatsachen darzustellen. Dabei ist der Effekt der Frauen mit hoher Bildung besonders augenfällig, widerspricht er doch der intuitiven Annahme, dass hohe Bildung per se die Chancen am Markt erhöht und damit von keinem Akteur unwahr dargestellt werden muss (vgl. auch Schmitz 2010). Geht man allerdings davon aus, dass Männer vor allem Partnerinnen suchen, die ein gleiches oder niedrigeres Bildungsniveau haben als sie selbst (Blossfeld/Timm 2003), dann bedeutet ein hohes Bildungsniveau eine massive Einengung des Partnermarktes. Frauen scheinen deswegen ihre hohe Bildung als Restriktion auf dem Partnermarkt wahrzunehmen. In dieser Kategorie waren, um dies in Erinnerung zu rufen, Personen mit einem (Fach-)Hochschulabschluss oder einer Promotion enthalten. Es ist zu vermuten, dass diese Frauen ihre zusätzlichen Bildungszertifikate eher verschweigen und z.B. auf die Angabe eines Doktortitels verzichten oder anstelle des Hochschulabschlusses lediglich das Abitur als formalen

15 Die Regressionskoeffizienten werden ebenso wie in der logistischen Regression mithilfe der Maximum-Likelihood-Methode geschätzt und können in ähnlicher Weise interpretiert werden (vgl. Powers/Xie 2000: 84). 
Bildungstitel angeben oder aber ganz auf die Angabe ihres Bildungsniveaus im Nutzerprofil verzichten.

Tabelle 2: Ergebnisse der komplementären Log-log-Regression zur unwahren Selbstdarstellung des Bildungsniveaus im Nutzerprofil

\begin{tabular}{|c|c|c|c|c|c|c|}
\hline & \multicolumn{3}{|c|}{ Männer } & \multicolumn{3}{|c|}{ Frauen } \\
\hline & $\begin{array}{c}\text { M1 } \\
b /(s e)\end{array}$ & $\begin{array}{c}\text { M2 } \\
b /(s e)\end{array}$ & $\begin{array}{c}\text { M3 } \\
b /(s e)\end{array}$ & $\begin{array}{c}\text { M1 } \\
b /(s e)\end{array}$ & $\begin{array}{c}\text { M2 } \\
\text { b/(se) }\end{array}$ & $\begin{array}{c}\text { M3 } \\
b /(s e)\end{array}$ \\
\hline \multicolumn{7}{|l|}{ spezifische Kompensation } \\
\hline \multirow[t]{2}{*}{ niedriges Bildungsniveau } & $1.08^{\star \star \star}$ & $0.75^{\star}$ & $0.73^{\star}$ & $1.17^{*}$ & 0.90 & $1.19^{*}$ \\
\hline & $(0.32)$ & $(0.34)$ & $(0.34)$ & $(0.56)$ & $(0.58)$ & $(0.58)$ \\
\hline mittleres Bildungsniveau & Ref. & Ref. & Ref. & Ref. & Ref. & Ref. \\
\hline \multirow[t]{2}{*}{ hohes Bildungsniveau } & $-0.90+$ & -0.78 & $-0.80+$ & $0.99^{*}$ & $1.19^{*}$ & $1.27^{\star \star}$ \\
\hline & $(0.48)$ & $(0.48)$ & $(0.48)$ & $(0.47)$ & $(0.48)$ & $(0.49)$ \\
\hline \multicolumn{7}{|l|}{ unspezifische Kompensation } \\
\hline \multirow[t]{2}{*}{ Alter } & & $-0.03^{*}$ & $-0.03^{*}$ & & -0.01 & -0.01 \\
\hline & & $(0.01)$ & $(0.01)$ & & $(0.02)$ & $(0.02)$ \\
\hline \multirow[t]{2}{*}{ BMI } & & -0.05 & -0.05 & & $0.07^{\star *}$ & $0.07^{* *}$ \\
\hline & & $(0.03)$ & $(0.04)$ & & $(0.02)$ & $(0.02)$ \\
\hline \multirow[t]{2}{*}{ Größe } & & $-0.03+$ & $-0.03+$ & & -0.04 & -0.05 \\
\hline & & $(0.02)$ & $(0.02)$ & & $(0.03)$ & $(0.03)$ \\
\hline \multicolumn{7}{|l|}{ ressourcenunabhäng. Variable } \\
\hline \multirow[t]{2}{*}{ Beziehungswunsch: feste Bez. } & & & $-0.55+$ & & & $-1.77^{\star \star *}$ \\
\hline & & & $(0.31)$ & & & $(0.43)$ \\
\hline \multirow[t]{2}{*}{ Konstante } & $-2.96^{\star \star \star}$ & $-2.92^{\star \star *}$ & $-2.48^{\star * *}$ & $-3.75^{\star * *}$ & $-4.25^{\star * \star}$ & $-3.23^{\star * *}$ \\
\hline & $(0.17)$ & $(0.18)$ & $(0.30)$ & $(0.33)$ & $(0.47)$ & $(0.51)$ \\
\hline Pseudo- $R^{2}(\mathrm{NK})$ & 0.05 & 0.08 & 0.09 & 0.04 & 0.09 & 0.18 \\
\hline Pseudo-R ${ }^{2}$ (MF) & 0.04 & 0.07 & 0.07 & 0.03 & 0.08 & 0.16 \\
\hline AIC & 0.40 & 0.39 & 0.39 & 0.32 & 0.32 & 0.30 \\
\hline unwahre Darstellung ja/nein & $54 / 996$ & $54 / 996$ & $54 / 996$ & $23 / 582$ & 23/582 & $23 / 582$ \\
\hline $\mathrm{N}$ & 1.050 & 1.050 & 1.050 & 605 & 605 & 605 \\
\hline
\end{tabular}

Signifikanz: ${ }^{+} 0,1 ; * \mathrm{p} \leq 0,05 ; * * \mathrm{p} \leq 0,01 ; * * * \mathrm{p} \leq 0,001$. Quelle: Online-Befragung von Nutzern einer deutschsprachigen Online-Kontaktbörse, eigene Berechnungen. Anmerkung: Alter, BMI (Body Mass Index) und Größe sind zentriert.

Modell 2 nimmt zusätzlich zum Bildungsniveau weitere Merkmale des Befragten auf, und zwar das Alter, den Body Mass Index und die Körpergröße. Damit möchten wir prüfen, ob die Akteure, unter Kontrolle ihrer Bildung, in Abhängigkeit von weiteren partnermarktrelevanten Merkmalen auf Strategien der Unwahrheit zurückgreifen. Wir prüfen also, ob sich Strategien der unspezifischen Kompensation beobachten lassen. In Modell 3 wird zusätzlich kontrolliert, ob der Befragte auf der Suche nach einer festen Beziehung ist. Im Folgenden wenden wir uns der Interpretation des Modells $3 \mathrm{zu}$, da sich die Ergebnisse durch Aufnahme der zusätzlichen Variable „fester Beziehungswunsch“ nicht wesentlich verändern (Unterschied der Modelle 2 und 3). Der Regressionskoeffizient der hohen Bildung bei Männern und der Koeffizient der niedrigen Bildung bei Frauen verfehlt in Modell 2 nur sehr knapp die Signifikanzgrenze von 10 Prozent und wird deshalb nicht inhaltlich interpretiert. Mit steigendem Alter der Männer verringert sich, bei Konstanthaltung des Bildungsniveaus, die Wahrscheinlichkeit in den Bildungsangaben im Nutzer- 
profil unehrlich zu sein. Von zwei Männern, die beide ein mittleres Bildungsniveau haben, hat also der ältere der beiden Männer eine geringere Wahrscheinlichkeit auf Unwahrheiten in seinem Bildungsniveau zurückzugreifen. Das Alter der Männer kann dabei als statusrelevante Ressource interpretiert werden, denn neben Bildungszertifikaten bestimmt auch das Alter des Mannes dessen Einkommenspotenzial (Oppenheimer 1988). Unter Bedingungen des traditionellen Modells des männlichen Ernährers werden Frauen daher stärker ältere Männer (bis zu einer gewissen Grenze) als jüngere Männer bevorzugen. Indem Männer diese Erwartung der Frauen an potenzielle Partner vorweg nehmen, kann das Auswirkungen auf ihre Neigung haben, auf Unwahrheiten in ihrer Selbstdarstellung zurückzugreifen.

Der Body Mass Index zeigt bei Männern, wie theoretisch erwartet, keinen signifikanten Einfluss, wohl aber die Körpergröße. Je größer ein Mann ist, desto geringer ist die Wahrscheinlichkeit, dass er sein Bildungsniveau den Tatsachen nicht entsprechend darstellt. Dieser Befund erhärtet unsere Annahme, dass die Akteure am Partnermarkt Stärken in einem Merkmal dazu nutzen, um vermeintliche oder tatsächliche Schwächen in einem anderen Merkmal auszugleichen. Gleichzeitig heißt das aber auch, dass eine geringe Körpergröße bei Männern, unter Kontrolle des Bildungsniveaus, die Wahrscheinlichkeit erhöht, in den Bildungsangaben zu lügen. Kleine Männer neigen im Vergleich zu großen Männern eher dazu, in ihrem Bildungsabschluss zu täuschen. Sie sind eher dazu gezwungen, ihre Aufmerksamkeits- und Kontaktchancen auch durch Unwahrheit hinsichtlich ihrer Bildung zu erhöhen, jedoch letztlich mit dem Risiko geringerer Tauschchancen in Form einer sich verfestigenden Beziehung. Damit greifen Akteure auch auf Unwahrheiten zurück, die nicht zwingend eine direkte Kompensation ihrer Merkmalsschwächen zum Gegenstand haben müssen. Es ist aber davon auszugehen, dass - um bei diesem Beispiel zu bleiben - ein sehr kleiner Mann auch auf Unwahrheiten in den Größenangaben und damit zusätzlich auf eine spezifische Kompensation dieser Merkmalsschwäche zurückgreift. Mit dem Alter und der Größe des Mannes haben wir damit für die Strategie der unspezifischen Kompensation zwei Beispiele aufgezeigt.

In Modell 3 der Frauen hat der Body Mass Index einen signifikant positiven Effekt auf die Wahrscheinlichkeit hinsichtlich des Bildungsabschlusses die Unwahrheit anzugeben. Körperlich attraktivere Frauen weisen also eine geringere Wahrscheinlichkeit auf, die Unwahrheit in ihrem Bildungsniveau anzugeben. Während Männer Nachteile in ihrem Bildungsniveau durch ihre Körpergröße und das Alter auszugleichen versuchen, ist bei Frauen die körperliche Attraktivität ein strategischer Ansatzpunkt, um vermeintliche oder tatsächliche Schwächen im Bildungsniveau zu kompensieren.

Schließlich bleibt noch die Interpretation des Effekts des Beziehungswunsches. Es zeigt sich, dass sowohl Männer als auch Frauen, die auf der Suche nach einer festen Beziehung sind, weniger dazu neigen, auf Unwahrheiten in ihrem Bildungsniveau zurückzugreifen. 
Geschlechts- und ressourcenspezifische Erklärung von Profiltäuschungen der körperlichen Attraktivität

Tabelle 3: Ergebnisse der komplementären Log-log-Regression zur unwahren Selbstdarstellung des Gewichts im Nutzerprofil

\begin{tabular}{|c|c|c|c|c|c|c|}
\hline & \multicolumn{3}{|c|}{ Männer } & \multicolumn{3}{|c|}{ Frauen } \\
\hline & $\begin{array}{c}\text { M1 } \\
b /(s e)\end{array}$ & $\begin{array}{c}\mathrm{M} 2 \\
\mathrm{~b} /(\mathrm{se})\end{array}$ & $\begin{array}{c}\text { M3 } \\
\text { b/(se) }\end{array}$ & $\begin{array}{c}\text { M1 } \\
\mathrm{b} /(\mathrm{se})\end{array}$ & $\begin{array}{c}\text { M2 } \\
\mathrm{b} /(\mathrm{se})\end{array}$ & $\begin{array}{c}\text { M3 } \\
b /(s e)\end{array}$ \\
\hline \multicolumn{7}{|l|}{ spezifische Kompensation } \\
\hline Gewicht & $\begin{array}{l}0.03^{\star \star *} \\
(0.00)\end{array}$ & $\begin{array}{l}0.03^{\star \star \star} \\
(0.00)\end{array}$ & $\begin{array}{l}0.03^{\star \star \star} \\
(0.00)\end{array}$ & $\begin{array}{l}0.03^{\star \star \star} \\
(0.00)\end{array}$ & $\begin{array}{l}0.03^{\star \star \star} \\
(0.01)\end{array}$ & $\begin{array}{l}0.03^{\star \star \star} \\
(0.01)\end{array}$ \\
\hline \multicolumn{7}{|l|}{ unspezifische Kompensation } \\
\hline Größe & & $\begin{array}{l}-0.01 \\
(0.01)\end{array}$ & $\begin{array}{l}-0.01 \\
(0.01)\end{array}$ & & $\begin{array}{l}-0.02 \\
(0.02)\end{array}$ & $\begin{array}{l}-0.02 \\
(0.02)\end{array}$ \\
\hline Alter & & $\begin{array}{l}-0.02^{*} \\
(0.01)\end{array}$ & $\begin{array}{l}-0.02^{*} \\
(0.01)\end{array}$ & & $\begin{array}{l}-0.00 \\
(0.01)\end{array}$ & $\begin{array}{l}-0.00 \\
(0.01)\end{array}$ \\
\hline niedriges Bildungsniveau & & $\begin{array}{l}0.79 \star \star \\
(0.27)\end{array}$ & $\begin{array}{l}0.78^{\star \star} \\
(0.27)\end{array}$ & & $\begin{array}{c}0.34 \\
(0.32)\end{array}$ & $\begin{array}{c}0.36 \\
(0.32)\end{array}$ \\
\hline mittleres Bildungsniveau & & Ref. & Ref. & & Ref. & Ref. \\
\hline hohes Bildungsniveau & & $\begin{array}{c}0.12 \\
(0.22)\end{array}$ & $\begin{array}{c}0.12 \\
(0.22)\end{array}$ & & $\begin{array}{l}0.85^{\star * *} \\
(0.24)\end{array}$ & $\begin{array}{l}0.85^{\star \star \star} \\
(0.24)\end{array}$ \\
\hline ressourcenunabhäng. Variable & & & & & & \\
\hline Beziehungswunsch: feste Bez. & & & $\begin{array}{l}-0.18 \\
(0.22)\end{array}$ & & & $\begin{array}{l}-0.38 \\
(0.26)\end{array}$ \\
\hline Konstante & $\begin{array}{l}-2.29 \star \star \star \\
(0.10)\end{array}$ & $\begin{array}{l}-2.39 * \star \star \\
(0.14)\end{array}$ & $\begin{array}{l}-2.24^{\star \star \star} \\
(0.23)\end{array}$ & $\begin{array}{l}-1.77^{\star \star \star} \\
(0.11)\end{array}$ & $\begin{array}{l}-2.17^{\star \star \star} \\
(0.20)\end{array}$ & $\begin{array}{l}-1.87^{\star \star \star} \\
(0.28)\end{array}$ \\
\hline Pseudo-R ${ }^{2}$ (NK) & 0.06 & 0.09 & 0.09 & 0.08 & 0.11 & 0.12 \\
\hline Pseudo-R ${ }^{2}$ (MF) & 0.04 & 0.07 & 0.07 & 0.05 & 0.08 & 0.08 \\
\hline AIC & 0.69 & 0.68 & 0.68 & 0.81 & 0.81 & 0.81 \\
\hline unwahre Darstellung ja/nein & $122 / 931$ & $122 / 931$ & $122 / 931$ & $92 / 515$ & $92 / 515$ & $92 / 515$ \\
\hline $\mathrm{N}$ & 1.053 & 1.053 & 1.053 & 607 & 607 & 607 \\
\hline
\end{tabular}

Signifikanz: ${ }^{+} 0,1 ; * \mathrm{p} \leq 0,05 ;{ }^{* *} \mathrm{p} \leq 0,01 ; * * * \mathrm{p} \leq 0,001$. Quelle: Online-Befragung von Nutzern einer deutschsprachigen Online-Kontaktbörse, eigene Berechnungen. Anmerkung: Gewicht, Größe und Alter sind zentriert.

Tabelle 3 enthält analog zu Tabelle 2 die Regressionsergebnisse zur unwahren Darstellung des Körpergewichts im Nutzerprofil. Sowohl Männer als auch Frauen weisen mit steigendem Gewicht eine höhere Wahrscheinlichkeit auf, in ihren Gewichtsangaben unehrlich zu sein. Vergleicht man die hier nicht dargestellten Konstanten des Nullmodells, zeigt sich, dass Frauen mit 15 Prozent im Vergleich zu Männern (12 Prozent) eine höhere Ausgangswahrscheinlichkeit haben, in ihrem Gewicht die Unwahrheit anzugeben. Dieses Ergebnis war bereits aus der deskriptiven Analyse in Tabelle 1 bekannt. Das Alter der Männer hat einen signifikant negativen Effekt. Unter Kontrolle des Körpergewichts haben wiederum jeweils ältere Männer eine geringere Wahrscheinlichkeit, in den Angaben zu ihrem Körpergewicht unehrlich zu sein. Bezüglich des Bildungsniveaus zeigt sich, dass niedrig gebildete Männer im Vergleich zu Männern mit mittlerem und hohem Bildungsniveau eine erhöhte Neigung haben, auf Unwahrheiten im Gewicht zurückzugreifen. Bei Frauen zeigt sich ein umgekehrter Bildungseffekt. Hier sind es die hoch gebildete Frauen, die im Vergleich zu Frauen mit mittlerem und niedrigem Bildungsniveau, unter Konstant- 
haltung des Gewichts, eine erhöhte Wahrscheinlichkeit haben, auf Unwahrheiten hinsichtlich des Gewichts zurückzugreifen. Für beide Geschlechter zeigt sich wieder ein negativer Effekt des Beziehungswunsches, der allerdings nicht signifikant ist.

Tabelle 4: Ergebnisse der komplementären Log-log-Regression zur unwahren Selbstdarstellung der Größe im Nutzerprofil

\begin{tabular}{|c|c|c|c|c|c|c|}
\hline & \multicolumn{3}{|c|}{ Männer } & \multicolumn{3}{|c|}{ Frauen } \\
\hline & $\begin{array}{c}\text { M1 } \\
\mathrm{b} /(\mathrm{se})\end{array}$ & $\begin{array}{c}M 2 \\
b /(s e)\end{array}$ & $\begin{array}{c}M 3 \\
b /(s e)\end{array}$ & $\begin{array}{c}\text { M1 } \\
b /(s e)\end{array}$ & $\begin{array}{c}\text { M2 } \\
b /(s e)\end{array}$ & $\begin{array}{c}M 3 \\
b /(s e)\end{array}$ \\
\hline \multicolumn{7}{|l|}{ spezifische Kompensation } \\
\hline Größe & $\begin{array}{l}-0.08^{\star \star \star} \\
(0.02)\end{array}$ & $\begin{array}{l}-0.08^{\star \star \star} \\
(0.02)\end{array}$ & $\begin{array}{l}-0.08^{\star \star \star} \\
(0.02)\end{array}$ & $\begin{array}{l}-0.02 \\
(0.04)\end{array}$ & $\begin{array}{l}-0.04 \\
(0.04)\end{array}$ & $\begin{array}{l}-0.05 \\
(0.04)\end{array}$ \\
\hline \multicolumn{7}{|l|}{ unspezifische Kompensation } \\
\hline Gewicht & & $\begin{array}{c}0.00 \\
(0.01)\end{array}$ & $\begin{array}{l}-0.00 \\
(0.01)\end{array}$ & & $\begin{array}{c}0.02+ \\
(0.01)\end{array}$ & $\begin{array}{c}0.02^{*} \\
(0.01)\end{array}$ \\
\hline Alter & & $\begin{array}{l}-0.02+ \\
(0.01)\end{array}$ & $\begin{array}{l}-0.02+ \\
(0.01)\end{array}$ & & $\begin{array}{l}-0.02 \\
(0.02)\end{array}$ & $\begin{array}{l}-0.02 \\
(0.02)\end{array}$ \\
\hline niedriges Bildungsniveau & & $\begin{array}{l}1.12^{\star \star \star} \\
(0.34)\end{array}$ & $\begin{array}{l}1.09^{\star \star} \\
(0.34)\end{array}$ & & $\begin{array}{l}-0.35 \\
(1.10)\end{array}$ & $\begin{array}{l}-0.09 \\
(1.10)\end{array}$ \\
\hline mittleres Bildungsniveau & & Ref. & Ref. & & Ref. & Ref. \\
\hline hohes Bildungsniveau & & $\begin{array}{l}-0.36 \\
(0.45)\end{array}$ & $\begin{array}{l}-0.40 \\
(0.45)\end{array}$ & & $\begin{array}{l}1.78^{\star \star \star} \\
(0.52)\end{array}$ & $\begin{array}{l}1.84^{\star \star \star} \\
(0.53)\end{array}$ \\
\hline ressourcenunabhäng. Variable & & & & & & \\
\hline Beziehungswunsch: feste Bez. & & & $\begin{array}{l}-1.09 * \star \star \\
(0.30)\end{array}$ & & & $\begin{array}{l}-1.92^{\star \star \star} \\
(0.48)\end{array}$ \\
\hline Konstante & $\begin{array}{l}-2.85^{\star \star *} \\
(0.14)\end{array}$ & $\begin{array}{l}-3.01^{\star * *} \\
(0.19)\end{array}$ & $\begin{array}{l}-2.20^{\star * *} \\
(0.27)\end{array}$ & $\begin{array}{l}-3.62^{* * *} \\
(0.38)\end{array}$ & $\begin{array}{l}-4.45^{\star \star \star} \\
(0.53)\end{array}$ & $\begin{array}{l}-3.32^{\star \star *} \\
(0.58)\end{array}$ \\
\hline Pseudo-R ${ }^{2}$ (NK) & 0.05 & 0.11 & 0.14 & 0.00 & 0.10 & 0.21 \\
\hline Pseudo-R ${ }^{2}$ (MF) & 0.04 & 0.09 & 0.12 & 0.00 & 0.09 & 0.19 \\
\hline AIC & 0.36 & 0.35 & 0.35 & 0.27 & 0.26 & 0.25 \\
\hline unwahre Darstellung ja/nein & 49/1.033 & 49/1.033 & 49/1.033 & $18 / 587$ & $18 / 587$ & $18 / 587$ \\
\hline $\mathrm{N}$ & 1.052 & 1.052 & 1.052 & 605 & 605 & 605 \\
\hline
\end{tabular}

Signifikanz: ${ }^{+} 0,1 ; * \mathrm{p} \leq 0,05 ; * * \mathrm{p} \leq 0,01 ; * * * \mathrm{p} \leq 0,001$. Quelle: Online-Befragung von Nutzern einer deutschsprachigen Online-Kontaktbörse, eigene Berechnungen. Anmerkung: Größe, Gewicht und Alter sind zentriert.

Abschließend zeigt Tabelle 4 die Regressionsergebnisse für die unwahre Selbstdarstellung der Körpergröße. Je größer ein Mann ist, umso geringer ist die Wahrscheinlichkeit, seine Körpergröße im Nutzerprofil unwahr darzustellen. Dieser Befund ist anschlussfähig an die Überlegungen zum Stellenwert männlicher Körpergröße auf dem Partnermarkt. Das Alter der Männer und der Beziehungswunsch zeigen wieder einen signifikant negativen Effekt. Weiterhin haben Männer mit niedrigem Bildungsniveau im Vergleich zu Männern mit mittlerem und hohem Bildungsniveau eine erhöhte Neigung, in ihrer Köpergröße unehrlich zu sein.

Bei Frauen zeigt sich, wie erwartet, kein signifikanter Effekt ihrer Körpergröße auf die Wahrscheinlichkeit, in diesem Merkmal unwahre Informationen bereitzustellen. Bemerkenswert ist der signifikant positive Effekt des Gewichts bei Frauen. Frauen scheinen nur dann unehrliche Angaben hinsichtlich ihrer Körpergröße zu machen, wenn sie etwas mehr Gewicht haben. Wenn Frauen also unehrliche Größenangaben machen, dann geht es 
in erster Linie nicht um ihre Körpergröße, sondern sie scheinen diese Angaben zu nutzen, um ein erhöhtes Gewicht in Relation zur Körpergröße besser kaschieren zu können. Dabei greifen Frauen, wenn ihnen das notwendig erscheint, auf eine Beschönigung ihres Gewichts zurück (vgl. Tabelle 3). Da aber diese spezifische Kompensation recht bald an die Grenzen der legitimen Optimierung stößt, zumindest wenn dem potenziellen Partner ein einigermaßen realistisches Bild vermittelt werden soll, greifen Frauen, um dies zusätzlich zu kompensieren, vermutlich auch auf Unwahrheiten in der Körpergröße und damit auf eine unspezifische Kompensation zurück. Und so ist es möglich, mit kleineren Unwahrheiten an verschiedenen Stellen die Gesamtattraktivität - also den Partnerwert - zu verbessern, ohne zu sehr von den Tatsachen abweichen zu müssen. Weiterhin zeigen sich wieder ein signifikant positiver Effekt des hohen Bildungsniveaus und ein signifikant negativer Effekt des Beziehungswunsches.

Zum Effekt des Beziehungswunsches ließen unsere theoretischen Überlegungen erwarten, dass die Nutzer der Kontaktbörse umso weniger auf Mittel der Täuschung zurückgreifen sollten, je fester ihr Beziehungswunsch ist. Dieser Befund zeigt sich auch: Über alle Modelle und beide Geschlechter hinweg ist die Wahrscheinlichkeit zu täuschen für diejenigen Akteure geringer, die einen Partner für eine festen Beziehung suchen. Allerdings ist dieser Befund hinsichtlich des Körpergewichts für beide Geschlechter nicht signifikant. Wir interpretieren diesen Effekt wie folgt: Das Bildungsniveau und die Körpergröße können als relativ stabile Merkmale wahrgenommen werden, weshalb die Partnersuchenden womöglich davon ausgehen, dass sie hinsichtlich dieser Merkmale nicht täuschen sollten. Bei einem festen Beziehungswunsch investieren Männer und Frauen deshalb weniger in ihre Aufmerksamkeitschancen durch die Optimierung dieser Merkmale in ihrem Profil. Demgegenüber ist das Körpergewicht ein Merkmal, das stärker beeinflussbar ist. Die Partnersuchenden könnten darauf spekulieren, dieses Merkmal bis zu einem realen Treffen mit dem potenziellen Partner tatsächlich noch etwas zu optimieren. Außerdem scheinen Beschönigungen im Gewicht vergleichsweise legitim zu sein (vgl. Toma et al. 2008); sie sind zudem - durch die recht feinen Optimierungsmöglichkeiten bei einem späteren persönlichen Treffen relativ schwierig zu entdecken. Wenn aber die Entdeckungswahrscheinlichkeit der Unwahrheit recht gering scheint, wird die Optimierung im Profil und damit die Investition in die Aufmerksamkeits- und Kontaktchancen umso lohnender.

\section{Zusammenfassung und Diskussion}

Das Ziel dieses Beitrags war die Untersuchung von Mustern unwahrer Selbstdarstellung auf Online-Kontaktbörsen. Dieser Beitrag grenzt sich dabei insofern von bisherigen Untersuchungen $a b$, die den Rückgriff auf Unwahrheiten und Täuschungen vorwiegend durch Persönlichkeitseigenschaften der Individuen erklärt haben (vgl. z.B. Hall et al. 2010; Phillips et al. 2011), als diese Untersuchung die relationale Position der Akteure am Partnermarkt berücksichtigt. Die Berücksichtigung der Position am Partnermarkt weist auf die Bedeutung der geschlechtsspezifischen Ressourcenausstattung der Akteure und der spezifischen Spielregeln am Partnermarkt hin. Die allgemeine Situation am digitalen Partnermarkt zeichnet sich durch eine hohe Konkurrenz um Aufmerksamkeits- und Kon- 
taktchancen aus: Alle Akteure sind dazu veranlasst, sich möglichst attraktiv und interessant zu präsentieren, um von potenziellen Partnern angeschrieben zu werden oder eine Antwort auf eine Kontaktofferte zu erhalten. Diejenigen Akteure, die jedoch relativ schlecht mit partnermarktrelevanten Ressourcen ausgestattet sind und damit eine relativ schlechte Position am Partnermarkt innehaben, müssen am ehesten auf Strategien der Unwahrheit zurückgreifen, um ihre Aufmerksamkeits- und Kontaktchancen zu erhöhen. Die theoretische Diskussion um die am Partnermarkt geltenden Konventionen im Hinblick auf die Quantität und Qualität der Unwahrheit hat uns dabei zur Annahme zweier Kompensationsstrategien geführt. Da anzunehmen ist, dass Akteure um die am Partnermarkt geltenden Konventionen wissen, scheint eine naheliegende Strategie zu sein - wenn schon auf Unwahrheiten zurückgegriffen werden muss - in verschiedenen Merkmalen im konventionell akzeptablen Maß, also überall nur im geringen Umfang zu täuschen und damit nie allzu sehr von den Tatsachen in einzelnen Merkmalen abzuweichen. Da jedoch einige Nutzer durch eine spezifische Unwahrheit im konventionell üblichen Maß ihren niedrigen Partnerwert und damit ihre geringen Aufmerksamkeitschancen nicht hinreichend kompensieren können, greifen diese auch auf unspezifische Kompensationen zurück. Diese Strategie, sich lediglich in kleinen Ausmaßen der Unwahrheit zu bedienen, scheint damit ein Ausweg aus dem Dilemma zu sein, sowohl die eigenen Aufmerksamkeits- und Kontaktchancen in der Masse potenzieller Partner zu erhöhen, ohne dabei auf der anderen Seite zu sehr die Chancen auf das letztliche Ziel der Etablierung einer Paarbeziehung zu verringern. Während der Mechanismus der spezifischen Kompensation, also der Unwahrheit dort wo der Nachteil vorliegt, bereits in anderen Untersuchungen zur strategischen Selbstdarstellung am Beispiel des Online-Datings nachgewiesen werden konnte (vgl. Hancock et al. 2007; Toma et al. 2008; Toma/Hancock 2010), ist die Berücksichtigung der relationalen Position der Akteure am Partnermarkt und der daraus abgeleiteten Idee der unspezifischen Kompensation als zusätzliche Ausgleichsstrategie unseres Wissens bislang nicht theoretisch konzipiert und empirisch untersucht worden.

Insgesamt zeigen unsere empirischen Analysen, dass der Rückgriff auf Unwahrheiten sowohl mit dem Geschlecht als auch mit der Ressourcenausstattung der Akteure variiert. Es sind gemäß unseren Erwartungen nicht alle Akteure, die die besonderen Kontextbedingungen auf Online-Kontaktbörsen nutzen, um sich unwahr darzustellen, sondern Akteure, die vermeintliche oder tatsächliche Nachteile in Merkmalen aufweisen von denen sie annehmen, dass das Gegengeschlecht diese an potenziellen Partnern wertschätzt. Unsere Analysen sind deshalb zugleich ein Hinweis darauf, welche Vorstellungen in den Köpfen von Frauen und Männern darüber bestehen, welche Erwartungen das andere Geschlecht an einen potenziellen Partner hat. Die Analysen haben gezeigt, dass aus Sicht der Männer Frauen vor allem statusrelevante Eigenschaften, wie sie im Bildungsniveau und Alter hervortreten, an potenziellen Partnern wertschätzen. Auch die Körpergröße des Mannes ist - zumindest in der Annahme der Männer - ein relevantes Auswahlkriterium der Frauen. Umgangssprachlich formuliert sind „Lügner“ hinsichtlich dieser Merkmalsdimension eher Männer, die kurze Beine haben. Aus Sicht der Frauen legen Männer vor allem auf Merkmale der physischen Erscheinung bei potenziellen Partnerinnen Wert. Auffällig ist dabei vor allem der über alle Modelle hinweg signifikante Effekt hoher Bildungsressourcen bei Frauen, der darauf hindeutet, dass Frauen die Erwartung haben, dass eine hohe Bildung - zumindest im Kontext der Partnerwahl - für sie nachteilig sein kann. 
Unsere Analysen zeigen, dass traditionelle Geschlechterrollen immer noch in den Köpfen von Männern und Frauen verankert zu sein und als Orientierung zu dienen scheinen.

Unsere empirischen Analysen zu Unwahrheiten im Bildungsniveau und in Merkmalen der physischen Attraktivität haben exemplarischen Charakter und können ebenso auf andere Bereiche, wie Unwahrheit im Beziehungswunsch und Unwahrheit bezüglich des Lebensalters erweitert werden. Täuschungen im Prozess der Paarbildung stellen zudem alltägliche Handlungen dar. So ist es schwer vorstellbar, dass Menschen eine Paarbeziehung mit einer Strategie der schonungslosen Offenheit zu realisieren vermögen (vgl. z.B. die Studie von Kaufmann 2004). Unwahrheiten können also - solange sie ein akzeptables Maß nicht überschreiten - sehr funktional sein. Weitere Untersuchungen müssten zeigen, ob und wie die beschriebenen Mechanismen und Strategien auch außerhalb des Internets in herkömmlichen Begegnungskontexten handlungswirksam werden.

\section{Literatur}

Agresti, A. (2002). Categorical data analysis. Hoboken, NJ: Wiley-Interscience ( $2^{\text {nd }}$ edition).

Barnes, J. A. (1994). A pack of lies. Towards a sociology of lying. Cambridge: University Press.

Ben-Ze'ev, A. (2004). Love online. Emotions on the Internet. Cambridge: University Press.

Biernat, M., Manis, M. \& Nelson, T. E. (1991). Stereotypes and standards of judgment. Journal of Personality and Social Psychology, 60, 4, S. 485-499.

Blau, P. M. (1964). Exchange and power in social life. New York: John Wiley \& Sons.

Blau, P. M. (1968). Social exchange. In: Sills, D. L. (Hrsg.), International enyclopedia of the social sciences. Volume 7. New York: Macmillan, S. 452-457.

Blossfeld, H.-P. \& Timm, A. (Hrsg.) (2003). Who marries whom? Educational systems as marriage markets in modern societies. Dordrecht: Kluwer Academic Publishers.

Blossfeld, H.-P. \& Drobnič, S. (Hrsg.) (2001). Careers of couples in contemporary societies. From male breadwinner to dual earner families. Oxford: Oxford University Press.

Blossfeld, H.-P. \& Timm, A. (1997). Der Einfluß des Bildungssystems auf den Heiratsmarkt. Eine Längsschnittanalyse der Wahl des ersten Ehepartners im Lebenslauf. Kölner Zeitschrift für Soziologie und Sozialpsychologie, 49, 3, S. 440-476.

Buss, D. M. \& Schmitt, D. P. (1993). Social strategies theory: An evolutionary perspective on human mating. Psychological Review 100, 2, S. 204-232.

Buss, D. M. \& Barnes, M. L. (1986). Preferences in human mate selection. Journal of Personality and Social Psychology 50, 3, S. 559-570.

Brym, R. J. \& Lenton, R. L. (2001). Love online: A report on digital dating in Canada. Toronto. http://projects.chass.utoronto.ca/brym/loveonline.pdf [Stand: 2011-02-21].

Butler-Smith, P., Cameron, S. \& Collins, A. (1998). Gender differences in mate search effort: An exploratory economic analysis of personal advertisements. Applied Economics 30, 10, S. 1277-1285.

Cameron, C., Oskamp, S. \& Sparks, W. (1977). Courtship American style: Newspaper ads. The Family Coordinator, 26, 1, S. 27-30.

Campos, L. de Sousa, Otta, E. \& Siqueira, J. de Oliveira (2002). Sex differences in mate selection strategies: Content analyses and responses to personal advertisements in Brazil. Evolution and Human Behavior 23, 5, S. 395-406.

Diekmann, A. (2008). Empirische Sozialforschung. Grundlagen, Methoden, Anwendungen. Reinbek bei Hamburg: Rowohlt (19. Auflage).

Donath, J. S. (1999). Identity and deception in the virtual community. In: Smith, M. \& Kollock, P. (Hrsg.), Communities in cyberspace. London: Routledge, S. 29-59.

Eagly, A. H. \& Wood, W. (1999). The origins of sex differences in human behavior. Evolved dispositions versus social roles. American Psychologist, 54, 6, S. 408-423. 
Edwards, J. N. (1969). Familial behavior as social exchange. Journal of Marriage and the Family 31, 3, S. $518-526$

Ekman, P. (1989). Weshalb Lügen kurze Beine haben. Über Täuschungen und deren Aufdeckungen im privaten und öffentlichen Leben. Berlin: de Gruyter.

Ellison, N., Heino, R. \& Gibbs, J. (2006). Managing impressions online: Self-presentation processes in the online dating environment. Journal of Computer-Mediated Communication, 11, 2, article 2.

England, P. \& McClintock, E. A. (2009). The gendered double standard of aging in US marriage markets. Population and Development Review, 35, 4, S. 797-816.

Esser, Hartmut (1999). Soziologie. Spezielle Grundlagen. Band 1: Situationslogik und Handeln. Frankfurt am Main/New York: Campus Verlag (3. Auflage).

Franzen, A. \& Hartmann. J. (2001). Die Partnerwahl zwischen Wunsch und Wirklichkeit: Eine empirische Studie zum Austausch von physischer Attraktivität und sozialem Status. In: Klein, T. (Hrsg.), Partnerwahl und Heiratsmuster. Sozialstrukturelle Voraussetzungen der Liebe. Opladen: Leske + Budrich, S. 183-206.

Gambetta, D. (2005). Deceptive mimicry in humans. In: Hurley, S. \& Chater, N. (Hrsg.), Perspectives on imitation: From neuroscience to social science. Volume 2: Imitation, human development, and culture. Cambridge, MA: The MIT Press, S. 221-241.

Gillis, J. S. \& Avis, W. E. (1980). The male-taller norm in mate selection. Personality and Social Psychology Bulletin 6, 3, S. 396-401.

Goffman, E. (2008). Wir alle spielen Theater. Die Selbstdarstellung im Alltag. München: Piper (6. Auflage).

Goffman, E. (1980 [orig. 1974]). Rahmen-Analyse. Ein Versuch über die Organisation von Alltagserfahrungen. Frankfurt am Main: Suhrkamp (1. Auflage).

Goffman, E. (1975 [orig. 1969]). Stigma. Über Techniken der Bewältigung beschädigter Identität. Frankfurt am Main: Suhrkamp (1. Auflage).

Goffman, E. (1969). The presentation of self in everyday life. Harmondsworth: Penguin Press (10. Auflage).

Hall, J. A., Park, N., Song, H. \& Mody, M. J. (2010). Strategic misrepresentation in online dating: The effects of gender, self-monitoring, and personality traits. Journal of Social and Personal Relationships 27, 1, S. 117-135.

Hancock, J. T., Toma, C. \& Elison, N. (2007). The truth about lying in online dating profiles. CHI 2007 Proceedings. (Paper for the conference on Computer-Human Interaction (CHI), San José, CA, April 28th to May 3th, 2007), S. 449-452.

Hassebrauck, M. (1990). Wer sucht wen? Eine inhaltsanalytische Untersuchung von Heirats- und Bekanntschaftsanzeigen. Zeitschrift für Sozialpsychologie, 21, 2, S. 101-122.

Harrison, A. A. \& Saeed, L. (1977). Let's make a deal: An analysis of revelations and stipulations in lonely hearts advertisements. Journal of Personality and Social Psychology 35, 4, S. 257-264.

Hettlage, R. (2003). Vom Leben in der Lügengesellschaft. In: Hettlage, R. (Hrsg.), Verleugnen, Vertuschen, Verdrehen. Leben in der Lügengesellschaft. Konstanz: UVK, S. 9-49.

Hill, P. B. \& Kopp, J. (2006). Familiensoziologie. Grundlagen und theoretische Perspektiven. Wiesbaden: VS Verlag für Sozialwissenschaften (4. Auflage).

Hirschman, E. C. (1987). People as products: Analysis of a complex marketing exchange. Journal of Marketing 51, 1, S. 98-108.

Hogan, B., Li, N. \& Dutton, W. H, (2011). A global shift in the social relationships of networked individuals: Meeting and dating online comes of age. Oxford: Oxford Internet Institute, Oxford University (Paper of the "Me, My Spouse and the Internet" project). http://blogs.oii.ox.ac.uk/couples/ [Stand: 2011-03-30].

Huston, T. L. \& Burgess, R. L. (1979). Social exchange in developing relationships: An overview. In: Burgess, R. L. \& Huston, T. L. (Hrsg.), Social exchange in developing relationships. New York: Academic Press, S. 3-28.

Kaufmann, J.-C. (2004). Der Morgen danach. Wie eine Liebesgeschichte beginnt. Konstanz: UVK. 
Kaupp, P. (1968). Das Heiratsinserat im sozialen Wandel - Ein Beitrag zur Soziologie der Partnerwahl. Stuttgart: Ferdinand Enke Verlag.

Kerckhoff, A. C. \& Davis. K. E. (1962). Value consensus and need complementary in mate selection. American Sociological Review 27, 3, S. 295-303.

Klein, T. \& Stauder, J. (2008). Partnermärkte in Deutschland im Spiegel eines neuen Erhebungsinstruments. In: Huinink, J. \& Feldhaus, M. (Hrsg.), Neuere Entwicklungen in der Beziehungs- und Familienforschung - Vorstudien zum Beziehungs- und Familienentwicklungspanel (PAIRFAM). Würzburg: Ergon, S. 77-114.

Lea, M. \& Spears, R. (1995). Love at first byte? Building personal relationships over computer networks. In: Wood, J. T. \& Duck, S. (Hrsg.), Under-studied relationships: Off the beaten track. Thousand Oaks, CA: Sage, S. 197-233.

Lenton, A. P. \& Stewart, A. (2008). Changing her ways: The number of options and mate-standard strength impact mate choice strategy and satisfaction. Judgment and Decision Making 3, 7, S. 501-511.

Lenz, K. (2009): Keine Beziehung ohne großes Theater. Zur Theatralität im Beziehungsaufbau. In: Willems, H. (Hrsg.), Theatralisierung der Gesellschaft. Band 1: Soziologische Theorie und Zeitdiagnose. Wiesbaden: VS Verlag für Sozialwissenschaften (1. Auflage), S. 239-258.

Lenz, K. (2006). Soziologie der Zweierbeziehung. Eine Einführung. Wiesbaden: VS Verlag für Sozialwissenschaften (3. Auflage).

Lenz, K. (2003). Täuschungen in Zweierbeziehungen. In: Hettlage, R. (Hrsg.), Verleugnen, Vertuschen, Verdrehen. Leben in der Lügengesellschaft. Konstanz: UVK, S. 65-96.

Long, S. J. (1997). Regression models for categorical and limited dependent variables. Thousand Oaks u.a.: Sage.

Lynn, M. \& Shurgot, B. A. (1984). Responses to lonely hearts advertisements: Effects of reported physical attractiveness, physique, and coloration. Personality and Social Psychology Bulletin, 10, 3, S. 349-357.

Mead, G. H. (1934). Mind, self and society from the standpoint of a social behaviorist. Chicago/London: University of Chicago Press.

Merkle, E. R. \& Richardson, R. A. (2000). Digital dating and virtual relating: Conceptualizing computer mediated romantic relationships. Family Relations 49, 2, S. 187-192.

Mulford, M., Orbell, J., Shatto, C. \& Stockard, J. (1998). Physical attractiveness, opportunity, and success in everyday exchange. American Journal of Sociology, 106, 6, S. 1565-1592.

Murstein, B. I. (1970). Stimulus - value - role: A theory of marital choice. Journal of Marriage and the Family, 32, 3, S. 465-481.

Murstein, B. I. (1972). Physical attractiveness and marital choice. Journal of Personality and Social Psychology, 22, 1, S. 8-12.

Murstein, B. I. (1986). Path to marriage. Beverly Hills: Sage.

Nyberg, D. (1993). The varnished truths: Truth telling and deceiving in ordinary life. Chicago: Chicago Press.

Oppenheimer, V. K. (1988). A theory of marriage timing. American Journal of Sociology, 94, 3, S. 563-591.

Ormell, J., Lindenberg, S., Steverink, N. \& Verbrugge, L. M. (1999). Subjective well-being and social production functions. Social Indicators Research. 46, 1, S. 61-90.

Pawlowski, B. \& Koziel, S. (2002). The impact of traits offered in personal advertisements on response rates. Evolution and Human Behavior 23, 2, S. 139-149.

Phillips, M., C., Meek, S. W. \& Vendemia, J. M. C. (2011). Understanding the underlying structure of deceptive behaviors. Personality and Individual Differences, 50, 6, S. 783-789.

Powers, D. A. \& Xie, Y. (2000). Statistical methods for categorical data analysis. San Diego: Academic Press.

Sautter, J. M., Tippett, R. M. \& Morgan, S. P. (2010). The social demography of Internet dating in the United States. Social Science Quarterly, 91, 2, S. 554-575.

Schmid, J. (2003). Über Techniken, andere hinters Licht zu führen. Sozialpsychologische Einblicke in das Repertoire von Täuschungen und Verzerrungen. In: Hettlage, R. (Hrsg.), Verleugnen, Vertuschen, Verdrehen. Leben in der Lügengesellschaft. Konstanz: UVK, S. 51-64.

Schmitz, A. (2010). Virtuelle Zwischengeschlechtlichkeit im Kontext relationaler Methodologie. Überlegungen zu einer Soziologie der digitalen Partnerwahl. In: Soeffner, H.-G. (Hrsg.), Unsichere Zei- 
ten. Herausforderungen gesellschaftlicher Transformationen. Verhandlungen des 34. Kongresses der Deutschen Gesellschaft für Soziologie in Jena 2008. Wiesbaden: VS Verlag für Sozialwissenschaften, S. 1-12 (CD-Rom, Ad-hoc-Gruppe 1 „Onlinedating“).

Schmitz, A. \& Skopek, J. (2011). Success in mating markets. A relational indicator of human mate value in E-Dating. Bamberg: University of Bamberg (Working paper of the project "Processes of Mate Choice in Online Dating").

Schmitz, A., Hofmann, K. \& Zillmann, D. (2010). Unehrlichkeit als Strategie. Eine qualitative Untersuchung am Beispiel der Selbstdarstellung im Online-Dating. Bamberg: Universität Bamberg (Arbeitspapier im Rahmen des Projektes „Prozesse der Partnerwahl auf Online-Kontaktbörsen“).

Schoen, R. \& Wooldredge, J. (1989). Marriage choices in North Carolina and Virginia, 1969-71 and 1979-81. Journal of Marriage and the Family, 51, 2, S. 465-481.

Schulz, F. \& Zillmann, D. (2009). Das Internet als Heiratsmarkt. Ausgewählte Aspekte aus Sicht der empirischen Partnerwahlforschung. Bamberg: Staatsinstitut für Familienforschung (ifb-Materialien 4/2009). www.ifb.bayern.de/imperia/md/content/stmas/ifb/materialien/mat_2009_4.pdf [Stand: 2011-03-30].

Schulz, F., Skopek, J., Klein, D. \& Schmitz, A. (2008). Wer nutzt Internetkontaktbörsen in Deutschland? Zeitschrift für Familienforschung/Journal of Family Research, 20, 3, S. 271-292.

Shepperd, J. A. \& Strathman, A. J. (1989). Attractiveness and height: The role of stature in dating preference, frequency of dating, and perceptions of attractiveness. Personality and Social Psychology Bulletin, 15, 4, S. 617-627.

Simmel, G. (1992 [orig. 1908]). Soziologie. Untersuchungen über die Formen der Vergesellschaftung. Band 11. Frankfurt am Main: Suhrkamp (1. Auflage).

Simon, H. A. (1956). Rational choice and the structure of the environment. Psychological Review 63, 2, S. 129-138.

Simon, H. A. (1990). Invariants of human behaviour. Annual Review of Psychology, 41, S. 1-19.

Skopek, J. (2011). Partnerwahl im Internet - Eine quantitative Analyse von Strukturen und Prozessen der Online-Partnersuche. Wiesbaden: VS Verlag für Sozialwissenschaften.

Skopek, J. (2010): Online-Panel: Auswertungen zum Antwortverhalten. Bamberg: Universität Bamberg (Arbeitspapier im Rahmen des Projektes „Prozesse der Partnerwahl auf Online-Kontaktbörsen").

Skopek, J., Schulz, F. \& Blossfeld, H.-P. (2009). Partnersuche im Internet. Bildungsspezifische Mechanismen bei der Wahl von Kontaktpartnern. Kölner Zeitschrift für Soziologie und Sozialpsychologie 61, 2, S. 183-210.

Stauder, J. (2008). Opportunitäten und Restriktionen des Kennenlernens. Zur sozialen Vorstrukturierung der Kontaktgelegenheiten am Beispiel des Partnermarkts. Kölner Zeitschrift für Soziologie und Sozialpsychologie 60, 2, S. 265-285.

Stevens, G., Owens, D. \& Schaefer, E. C. (1990). Education and attractiveness in marriage choices. Social Psychology Quarterly, 53, 1, S. 62-70.

Todd, P. M. \& Miller, G. F. (1999). From pride and prejudice to persuasion - Satisficing in mate search. In: Gigerenzer, G., Todd, P. M. \& ABC Research Group (Hrsg.), Simple heuristics that make us smart. New York: Oxford University Press, S. 287-308.

Toma, C. \& Hancock, J. T. (2010). Looks and lies: The role of physical attractiveness in online dating self-presentation and deception. Communication Research, 37, 3, S. 335-351.

Toma, C., Hancock, J. T. \& Ellison, N. (2008). Separating fact from fiction: Deceptive self-presentation in online dating profiles. Personality and Social Psychology Bulletin, 34, 8, S. 1023-1036.

Tovée, M. J., Hancock, P. J. B., Mahmoodi, S., Singleton, B. R. R. \& Cornelissen, P. L. (2002). Human female attractiveness: waveform analysis of body shape. Proceedings of the Royal Society of London, Series B: Biological Sciences, 269, (1506), S. 2205-2213.

Tovée, M.. J., Maisey, D, S., Emery, J. L. \& Cornelissen, P. L. (1999). Visual cues to female physical attractiveness. In: Proceedings of the Royal Society of London, Series B: Biological Sciences, 266 (1415), S. 211-218.

Tovée, M. J., Reinhardt, S., Emery, J. L. \& Cornelissen, P. L. (1998). Optimum body-mass index and maximum sexual attractiveness. The Lancet, 352, 15, S. 548. 
Eingereicht am/Submitted on: 18.04.2011

Angenommen am/Accepted on: 04.10.2011

Addresses of the authors/Anschriften der Autorin und der Autoren:

Dipl.-Soz. Doreen Zillmann (Korrespondenzautorin/Corresponding author)

Andreas Schmitz, M.A.

Prof. Dr. Dr. h.c. Hans-Peter Blossfeld

Otto-Friedrich-Universität Bamberg

Lehrstuhl für Soziologie I

Wilhelmsplatz 3

96047 Bamberg

Germany/Deutschland

E-Mail: doreen.zillmann@uni-bamberg.de andreas.schmitz@uni-bamberg.de hans-peter.blossfeld@uni-bamberg.de

\section{Anhang}

Tabelle A.1: Beschreibung der Analysestichprobe (Spaltenprozente bzw. Mittelwerte)

\begin{tabular}{|c|c|c|c|c|}
\hline & Männer & Frauen & Gesamt & $\mathbf{N}$ \\
\hline \multicolumn{5}{|l|}{ Geschlecht } \\
\hline männlich & - & - & 63,89 & 1.350 \\
\hline weiblich & - & - & 36,11 & 763 \\
\hline keine Angabe & - & - & - & - \\
\hline Durchschnittsalter (SD) & $40,29(12,31)$ & $42,34(12,43)$ & $41,03(12,39)$ & 2.107 \\
\hline \multicolumn{5}{|l|}{ Alter } \\
\hline unter 20 Jahre & 2,15 & 2,36 & 2,22 & 47 \\
\hline $20-29$ Jahre & 20,96 & 17,96 & 19,88 & 420 \\
\hline 30 - 39 Jahre & 24,81 & 19,00 & 22,72 & 480 \\
\hline 40 - 49 Jahre & 27,63 & 29,75 & 28,40 & 600 \\
\hline 50 - 59 Jahre & 17,56 & 22,94 & 19,50 & 412 \\
\hline 60 - 69 Jahre & 5,63 & 7,08 & 6,15 & 130 \\
\hline 70 - 79 Jahre & 0,96 & 0,66 & 0,85 & 18 \\
\hline 80 Jahre und älter & - & - & - & - \\
\hline keine Angabe & 0,30 & 0,26 & 0,28 & 6 \\
\hline \multicolumn{5}{|l|}{ Bildungsniveau } \\
\hline noch Schüler & 1,11 & 0,39 & 0,85 & 18 \\
\hline keinen Schulabschluss & 3,48 & 2,88 & 3,27 & 69 \\
\hline Haupt-/(Volks-)schulabschluss & 23,48 & 14,68 & 20,30 & 429 \\
\hline Mittlere Reife & 24,67 & 34,47 & 28,21 & 596 \\
\hline Fachhochschulreife & 12,81 & 11,93 & 12,49 & 264 \\
\hline Abitur & 17,63 & 20,45 & 18,65 & 394 \\
\hline anderer Schulabschluss & 1,11 & 0,26 & 0,80 & 17 \\
\hline keine Angabe & 15,70 & 14,94 & 15,43 & 326 \\
\hline
\end{tabular}




\begin{tabular}{|c|c|c|c|c|}
\hline & Männer & Frauen & Gesamt & $\mathbf{N}$ \\
\hline \multicolumn{5}{|l|}{ Ausbildungsniveau } \\
\hline noch in Ausbildung & 3,48 & 4,06 & 3,69 & 78 \\
\hline keinen Ausbildungsabschluss & 7,26 & 8,78 & 7,81 & 165 \\
\hline beruflicher Ausbildungsabschluss & 52,37 & 49,02 & 51,16 & 1.081 \\
\hline Fachhochschulabschluss & 9,56 & 11,14 & 10,13 & 214 \\
\hline Hochschulabschluss & 9,33 & 10,09 & 9,61 & 203 \\
\hline Promotion & 1,33 & 1,18 & 1,28 & 27 \\
\hline anderer beruflicher Abschluss & 0,30 & 0,66 & 0,43 & 9 \\
\hline keine Angabe & 16,37 & 15,07 & 15,90 & 336 \\
\hline \multicolumn{5}{|l|}{ Kombination Bildung \& Ausbildung } \\
\hline niedrig (ohne Schulab., HSO, MRO) & 7,70 & 9,17 & 8,23 & 174 \\
\hline mittel (HSM, MRM, ABIO, ABIM) & 55,04 & 51,77 & 53,86 & 1.138 \\
\hline hoch (FH, UNI, Promotion) & 18,37 & 20,31 & 19,07 & 403 \\
\hline keine Angabe & 18,89 & 18,74 & 18,84 & 398 \\
\hline \multicolumn{5}{|l|}{ Familienstand } \\
\hline ledig & 60,15 & 40,10 & 52,91 & 1.118 \\
\hline verheiratet & 3,70 & 0,66 & 2,60 & 55 \\
\hline verheiratet, aber getrennt lebend & 10,37 & 11,40 & 10,74 & 227 \\
\hline geschieden & 23,04 & 40,63 & 29,39 & 621 \\
\hline verwitwet & 2,74 & 7,21 & 4,35 & 92 \\
\hline keine Angabe & - & - & - & - \\
\hline \multicolumn{5}{|l|}{ Paarbeziehung } \\
\hline ohne Paarbeziehung & 89,78 & 92,53 & 90,77 & 1.918 \\
\hline mit Paarbeziehung (ohne Ehe) & 6,52 & 6,82 & 6,63 & 140 \\
\hline Ehe & 3,70 & 0,66 & 2,60 & 55 \\
\hline keine Angabe & - & - & - & - \\
\hline \multicolumn{5}{|l|}{ Kinder } \\
\hline Kinder vorhanden & 37,48 & 63,56 & 46,90 & 991 \\
\hline kinderlos & 62,52 & 36,44 & 53,10 & 1.122 \\
\hline keine Angabe & - & - & - & - \\
\hline \multicolumn{5}{|l|}{ Beziehungswunsch: feste Beziehung } \\
\hline trifft nicht zu & 7,56 & 4,85 & 6,58 & 139 \\
\hline teils/teils & 10,74 & 13,24 & 11,64 & 246 \\
\hline trifft zu & 75,85 & 78,24 & 76,72 & 1.621 \\
\hline keine Angabe & 5,85 & 3,67 & 5,06 & 107 \\
\hline Durchschnittsgröße in cm (SD) & $179,21(7,56)$ & $167,30(6,57)$ & $174,89(9,21)$ & 1.917 \\
\hline Durchschnittsgewicht in $\mathrm{kg}(S D)$ & $82,61(16,00)$ & $74,31(17,65)$ & $79,62(17,08)$ & 1.909 \\
\hline Durchschnittlicher Body Mass Index (SD) & $25,66(4,46)$ & $26,53(6,13)$ & $25,98(5,14)$ & 1.908 \\
\hline
\end{tabular}

Anmerkungen: ohne Schulab. = ohne Schulabschluss; HSO = Hauptschule ohne beruflichen Ausbildungsabschluss; MRO = Mittlere Reife ohne beruflichen Ausbildungsabschluss; HSM = Hauptschule mit beruflichem Ausbildungsabschluss; MRM = Mittlere Reife mit beruflichem Ausbildungsabschluss; $\mathrm{ABIO}=$ Abitur ohne beruflichen Ausbildungsabschluss; ABIM = Abitur mit beruflichem Ausbildungsabschluss; FM = Fachhochschulabschluss; UNI = Hochschulabschluss.

Quelle: Online-Befragung von Nutzern einer großen deutschen Online-Kontaktbörse; $\mathrm{N}=2.113$; eigene Berechnungen. 